\title{
The Demography of Super-Massive Black Holes: Growing Monsters at the Heart of Galaxies
}

\author{
Francesco Shankar \\ Max-Planck-Institut für Astrophysik, Karl-Schwarzschild-Str. 1, D-85748, Garching, \\ Germany
}

\begin{abstract}
Supermassive black holes (BHs) appear to be ubiquitous at the center of all galaxies which have been observed at high enough sensitivities and resolution with the Hubble Space Telescope. Their masses are found to be tightly linked with the masses and velocity dispersions of their host galaxies. On the other hand, BHs are widely held to constitute the central engines of quasars and active galactic nuclei (AGN) in general. It is however still unclear how BHs have grown, and whether they have co-evolved with their hosts. In this Review I discuss how, in ways independent of specific models, constraints on the growth history of BHs and their host galaxies have been set by matching the statistics of local BHs to the emissivity, number density, and clustering properties of AGNs at different cosmological epochs. I also present some new results obtained through a novel numerical code which evolves the $\mathrm{BH}$ mass function and clustering adopting broad distributions of Eddington ratios. I finally review $\mathrm{BH}$ evolution in a wider cosmological context, connecting $\mathrm{BH}$ growth to galaxy evolution.
\end{abstract}

Key words: black holes, supermassive; quasars; galaxies, active; statistics

\section{Introduction}

Supermassive black holes (BHs) are common at the center of most, if not all, galaxies (e.g., Magorrian et al., 1998; Ferrarese and Merritt, 2000; Gebhardt et al., 2000). As it will be discussed below, their mass is tightly

Email address: shankar@mpa-garching.mpg.de (Francesco Shankar) 
linked with several properties of their host galaxies. However, it still constitutes a major challenge understanding how these BHs have grown, and if they followed the mass assembly history of their host galaxies.

The discovery of such strong correlations lent further support to the seminal idea, already proposed more than forty years back (e.g., Salpeter, 1964; Lynden-Bell, 1969), that quasars and, more generally, active galactic nuclei (AGNs) are powered by mass accretion onto central BHs, which should now lie at the center of galaxies as relics of a past luminous phase. The only process in fact able to generate the high and highly concentrated powers observed in quasars is the release of gravitational energy from an infalling body of mass $m$ onto an extremely compact object of mass $M$. This process can then generate energies up to a maximum of $E=G M m / 5 R_{g}=0.1 \mathrm{mc}^{2}$, with the Schwarzschild radius $R_{g}=2 G M / c^{2} \sim 3 \times 10^{13}\left(M / 10^{8} \mathrm{M}_{\odot}\right) \mathrm{cm}$. Gravitational processes can therefore release significant fractions of the restmass energy, orders of magnitude more efficiently than the energy release from stellar nuclear reactions. For example, a quasar with luminosity of $L=10^{46} \mathrm{erg} \mathrm{s}^{-1}$ can be easily generated by a massive object of $10^{8} M_{\odot}$ confined within a region of size $\leq 10^{15} \mathrm{~cm}$.

The detection of any massive dark object at the center of a currently inactive galaxy is performed by analyzing the motion of gravitating bodies embedded in the strong $\mathrm{BH}$ gravitational field. In any given galaxy, a $\mathrm{BH}$ detection can be roughly labeled in terms of $r_{G}$, the radius of the sphere of influence within which the $\mathrm{BH}$ dominates the gravitational potential of its host galaxy. However, it is easily seen that for a $10^{8} M_{\odot}$, at a distance of $20 \mathrm{Mpc}, r_{G}$ is only 0.11 arcsec (e.g., Barth, 2004), which proves that reliable direct dynamical $\mathrm{BH}$ mass measurements can only be safely obtained in nearby galaxies. In particular, the presence of $\mathrm{BHs}$ in inactive galaxies is tested through the velocity dispersion of stars, water masers, and gas (see Ferrarese and Ford 2005). The possibly most exciting example is the detection of a $\mathrm{BH}$ at the center of our own Galaxy, which also represents one of the tightest constraints on the existence of a BH. Monitoring has been carried out for more than 10 years on 40 stars orbiting around the center of the Galaxy. Measurements in the infrared with adaptive optics imaging of the $10 \mathrm{~m}$ class telescopes have then allowed determining the three dimensional, Keplerian orbits of stars within ten light hours of the compact radio source at the center of the Milky Way (see Figure 1). A few of them have already passed the pericenter allowing Genzel and Karas (2007, and references therein) and Ghez et al. (2003) to measure a central mass of $\sim 3 \times 10^{6} M_{\odot}$. 

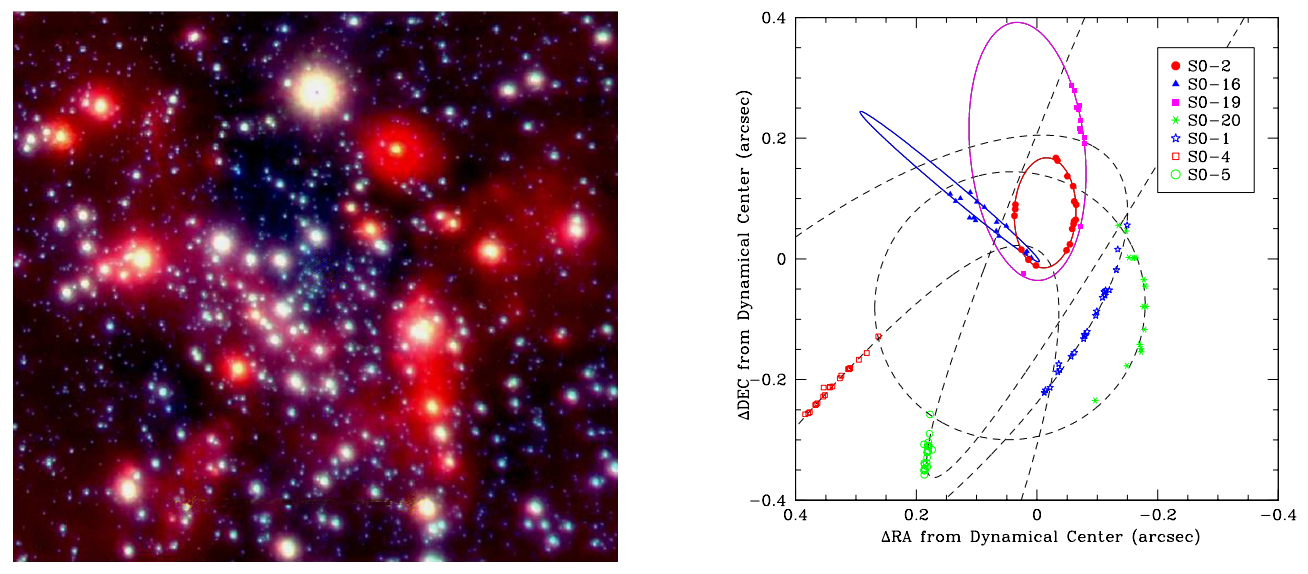

Figure 1: Left panel: a diffraction limited image of Sagittarius $\mathrm{A}^{*}(\sim 0.05$ arcsec resolution) from the $8 \mathrm{~m}$ ESO Very Large Telescope, taken with the NACO AO-camera and an infrared wavefront sensor at $1.6 / 2.2 / 3.7 \mu \mathrm{m}$. The central black hole is located in the centre of the box. NACO is a collaboration between ONERA (Paris), Observatoire de Paris, Observatoire Grenoble, MPE (Garching), and MPIA (Heidelberg). Right panel: positions on the sky as a function of time for the central stars orbiting the compact radio source Sagittarius $\mathrm{A}^{*}$. The data are from the UCLA group working with the Keck telescope. Figures taken from Genzel and Karas (2007) and references therein.

BHs are currently measured in active galaxies too. The first attempts used direct spectral fitting of the Big Blue Bump, the optical/UV/soft Xray continuum thermal emission from an optically thick, geometrically think accretion disk. More secure mass determinations are currently based on the Reverberation Mapping technique and extrapolations of the latter (see Ferrarese and Ford 2005 for a full description of such methods).

Given the overwhelming evidence for a strong connection between BHs and their hosts, in particular all massive galaxies have a central massive $\mathrm{BH}$, most theoretical models of galaxy formation now include recipes to grow BHs within galaxies (e.g., Granato et al., 2004; Di Matteo et al., 2005; Vittorini et al., 2005; Bower et al., 2006; Croton et al., 2006; Hopkins et al., 2006a; Lapi et al., 2006; Malbon et al., 2007; Monaco et al., 2007; Menci et al., 2004, 2006, 2008; Cook et al., 2009). However, as explained below, models differ significantly in the physical recipes adopted to evolve $\mathrm{BHs}$, and although sometimes the observables reproduced are the same, the underlying physics might be widely different.

It is therefore of extreme importance to probe $\mathrm{BH}$ evolution in ways independent of specific models. In this Review I will show how along the last 
years, several groups have been able to develop and refine semi-empirical techniques to constrain $\mathrm{BH}$ evolution from first principles. The Review will focus on the latest statistical results on the BH/AGN population and show how these information can be interconnected to derive interesting and firm constraints on how BHs must have evolved along cosmic time.

This Review will therefore not discuss details of BH/AGN emission processes (see the seminal paper by Rees 1984), nor the problem of seeding galaxies with BHs (e.g., Haiman and Quataert 2004), nor the problem of AGN unification models (e.g., Urry and Padovani, 1995; Maraschi, 2002; Elitzur, 2008, and references therein). On the other hand, it will cover aspects of $\mathrm{BH}$ evolution which have not been properly discussed before in a coherent framework.

In $\S 2$ all the main correlations which have been measured between $\mathrm{BH}$ mass and host galaxy properties will be discussed, putting more emphasis on the most recent empirical results still not covered by previous reviews on the subject. In $\S 3$ it will be shown how to measure the comoving number of BHs, and $\S \S 4$, 5, and 6 will discuss why the latter information represents an invaluable tool to understand how BHs have grown to the population we observe today. $\S 7$ will instead discuss how quasar clustering provides many, useful and independent constrains on $\mathrm{BH}$ evolution. A brief overview of how current models of galaxy formation are currently treating $\mathrm{BH}$ evolution, will be given in $\S 8$. This aspect has not yet been systematically addressed by previous reviews on BHs, and it is useful to show how current open problems in galaxy-BH co-evolution models can be naturally and simply addressed via the techniques outlined in this Review. The main conclusions of this whole discussion will be listed in $\S$.

\section{The tight relation between Black Holes and their host galaxies in the local Universe}

The presence of nuclear BHs in the majority of galaxies (or at least bulges) has become an accepted paradigm. The nuclear kinematics of all nearby galaxies for which data of the necessary depth and spatial resolution have been collected, show the clear signature of central mass concentrations beyond what can be attributed to known stellar populations. The implied BH masses $M_{\mathrm{bh}}$ are found to correlate with several global properties of the host galaxies, including the mass, $M_{\text {bulge }}$ (or luminosity $L_{\text {bulge }}$ ), velocity dispersion, $\sigma$, and light concentration $c_{\text {bulge }}$ of the spheroidal component 
(e.g. Ferrarese and Merritt, 2000; Gebhardt and et al., 2000; Graham et al., 2001; Marconi and Hunt, 2003; Häring and Rix, 2004), and the mass of the surrounding dark matter halo (e.g., Ferrarese, 2002a). In view of the clear importance of these correlations, it is worth discussing them below in some detail before progressing further in the Review.

\subsection{The Correlation between Black Hole mass and the luminosity/mass of} the bulge

Kormendy and Richstone (1995) reviewed the status of BH demography up to that time. Using a sample of only 8 well measured BH mass measurements they pointed out a tight correlation, with the BH mass being $2-3 \%$ of the stellar mass of the host. Magorrian et al. (1998) constructed 2D dynamical models for a sample of 36 nearby galaxies finding that the models required at the $95 \%$ confidence level the mass of the $\mathrm{BH}$ to be linearly correlated with the mass of their hosts as $M_{\mathrm{bh}} \sim 0.006 M_{\mathrm{star}}$. Updating the catalog of $\mathrm{BH}$ detections, Kormendy et al. (2000) concluded that the $\mathrm{BH}$ mass correlates much better with bulge luminosity than with the total luminosity of the galaxy, and that $\mathrm{BH}$ mass correlates with the luminosity of the high-density central component in disk galaxies independent of whether that component is a classical bulge (essentially a mini-elliptical) or a "pseudobulge" (believed to form via inward transport of disk material). McLure and Dunlop (2001) were the first to point out that if the sample is restricted to elliptical galaxies, the scatter is further significantly reduced. Despite the strong improvements in bulge-bar-disk decompositions (e.g., Peng et al., 2002; Gadotti, 2008; Laurikainen et al., 2009), due to the still small sample of local galaxies with secure $\mathrm{BH}$ mass measurements, it is still not clear whether the reduced scatter in ellipticals is mainly due to uncertainties associated in determining bulge masses in later-type galaxies, or to an intrinsic physical effect (see also discussions in Ferrarese and Ford 2005). McLure and Dunlop (2001) also showed that both active and inactive galaxies follow similar correlations both in slope and normalization, although the intrinsic scatter around the correlations for inactive galaxies is much lower.

Marconi and Hunt (2003) used $K$-band images from the 2MASS database, and performed an accurate bulge/disk decomposition for the nearby quiescent spiral galaxies with dynamically measured masses. They confirmed a decrease of the intrinsic scatter around the correlations when only earlytype galaxies were considered. However, their total $K$-band magnitudes were derived by extrapolating the image profiles to very large distances on 
implying an average increase of brightness of about 0.5 magnitudes (e.g., Batcheldor et al., 2007).

Graham (2007) critically revisited all the differing $M_{\mathrm{bh}}-L$ relations discussed above by McLure \& Dunlop, Marconi \& Hunt and others. He considered several previously not properly addressed issues finding that, when these adjustments are consistently applied to all the previous samples, results in relations which predict similar BH masses. His final result in the $K$-band reads

$$
\log \left(M_{\mathrm{bh}} / M_{\odot}\right)=(-0.37 \pm 0.04)\left(M_{K}+24\right)+8.29 \pm 0.08
$$

with an intrinsic scatter of 0.33 dex.

Gultekin et al. (2009) recently recomputed the relations between BH and host-galaxy bulge velocity dispersion and luminosity, using the updated sample of $49 M_{\mathrm{bh}}$ measurements and also adopting 19 upper limits. Restricting to early-type galaxies, they find

$$
\log \left(M_{\mathrm{bh}} / M_{\odot}\right)=(8.95 \pm 0.11)+(1.11 \pm 0.18) \times \log \left(L_{V} / 10^{11} L_{\odot, V}\right),
$$

with an intrinsic scatter of $0.38 \pm 0.09$ dex. The Authors claim their results to be insensitive to a wide range of assumptions about the measurement uncertainties and the distribution of intrinsic scatter. They also discuss how restricting the sample to the objects with resolution of the BH's sphere of influence might bias the results, a conclusion at variance with previous studies (e.g., Marconi and Hunt, 2003; Ferrarese and Ford, 2005).

Häring and Rix (2004) approached the correlation between BH mass and host stellar mass in a way independent of photometric measurements, i.e., utilizing a total of 30 galaxies with bulge masses derived through Jeans modeling or adopted from dynamical models in the literature. They found $M_{\mathrm{bh}} \sim 1.4 \times 10^{-3} M_{\text {bulge }}$ with an observed scatter of $\lesssim 0.30$ dex, a fraction of which attributed to measurement errors. Their results confirm and refine the previous work of Marconi and Hunt (2003).

In sum, all of the most recent and detailed investigations have settled to define a closely linear correlation between $\mathrm{BH}$ mass and host galaxy mass/luminosity over several decades, with a factor of proportionality of $2 \times 10^{-3}$, e.g., $M_{\mathrm{bh}} \simeq 2 \times 10^{-3} M_{\text {bulge }}$.

\subsection{The black hole mass velocity dispersion correlation}

The tight correlation between $\mathrm{BH}$ mass and the velocity dispersion $\sigma$ of the host galaxy is probably the correlation which received most atten- 
tion in the literature as it provides a direct proof of the strong link between BH mass and potential well of the galaxy. It was first pointed out by Ferrarese and Merritt (2000); Gebhardt and et al. (2000); Tremaine et al. (2002), although with some discrepancies in the basic results (see also Marconi et al. 2004), and we refer to those papers and to the review by Ferrarese and Ford (2005) for extensive discussions on this topic.

One of the latest determinations of the $M_{\mathrm{bh}}-\sigma$ obtained by Tundo et al. (2007) yields the result

$$
\log \left(M_{\mathrm{bh}} / M_{\odot}\right)=(8.21 \pm 0.06)+(3.83 \pm 0.21) \log \left(\sigma / 200 \mathrm{~km} \mathrm{~s}^{-1}\right)
$$

with an intrinsic scatter of $0.22 \pm 0.06$ dex. These Authors also point out that the $\sigma$ - $L$ relation of local early-type galaxies, including those having $\mathrm{BH}$ mass measurements, is different from the average $\sigma$ - $L$ relation of early type galaxies calibrated in the Sloan Digital Sky Survey (SDSS). This difference, they conclude, might bias the local BHMF. Lauer et al. (2007a) also stress that the curvature in the $\sigma-L$ relation for massive early-type galaxies implies that the $M_{\mathrm{bh}}-\sigma$ relation is unlikely to be a single power law. On similar lines, Wyithe (2006) suggested through a Bayesian analysis that a quadratic fit is a better description of the correlation between $\mathrm{BH}$ mass and $\sigma$ in the Tremaine et al. (2002) sample. $\mathrm{Hu}(2008)$ reinvestigated in detail the $M_{\mathrm{bh}}-\sigma$ relation, finding similar results to Eq. (3) for the ellipticals, but also pointing out that pseudo-bulges and core early-type galaxies seem to show significant departures from it, a possible signature of their different formation histories.

\subsection{Redshift Evolution of the correlations}

Both the local characterization, and the cosmic evolution of the above mentioned scaling relations are subject to intense observational studies (e.g., Shields et al., 2006; Peng et al., 2006; Lauer et al., 2007b; Treu et al., 2007; Shankar et al., 2009a), as they provide powerful constraints on theoretical models for the co-evolution of galaxies and BHs (e.g., Granato et al., 2004; Vittorini et al., 2005; Hopkins et al., 2006a; Croton et al., 2006; De Lucia et al., 2006; Cavaliere and Menci, 2007; Monaco et al., 2007; Marulli et al., 2008).

Treu et al. (2007) and Woo et al. (2008) have randomly compiled from the SDSS Data Release 4 a sample of about 20 Seyfert galaxies in the redshift range $0.37 \lesssim z \lesssim 0.57$. Shen et al. (2008) estimated the $M_{\mathrm{bh}}-\sigma$ relation for a larger sample of active galaxies up to $z=0.452$. While the latter claim that no significant evolution in the $M_{\mathrm{bh}}-\sigma$ relation is detectable from their 
sample, Woo et al. (2008) confirm the results by Treu et al. (2007) that a significant increase of $\sim 0.2$ dex in $\mathrm{BH}$ mass at fixed velocity dispersion must occur within $z=0$ and $z \sim 0.5$. However, systematic uncertainties may affect these estimates; for example, as also discussed by Woo et al. (2008), especially in galaxies with lower $\mathrm{BH}$ mass the host galaxy contribution to the $5100 \AA$ luminosity may lead to an overestimation of the true $\mathrm{BH}$ mass.

Peng et al. (2006) collected a sample of 31 lensed and 18 unlensed AGNs at redshifts $z>1.7$. They measured rest-frame R-band luminosities from $\mathrm{H}$-band fluxes and $\mathrm{BH}$ masses by applying virial relations based on emission line widths. They found that the BH-to-host galaxy luminosity at $z \sim 2$ is about the same as that at $z \sim 0$. Therefore, once the observed restframe luminosity has dimmed through substantial evolution to $z \sim 0$, at fixed $\mathrm{BH}$ mass the ratio BH-to-host luminosity grows significantly, and the resulting $\mathrm{BH}$-luminosity normalization is several times higher than the local one. Similar results were derived by McLure et al. (2006), who measured the $\mathrm{BH}$-to-host galaxy mass ratio in a sample of radio-loud AGNs in the redshift range $0<z<2$ finding $M_{\mathrm{bh}} / M_{\text {star }} \propto(1+z)^{2}$. Shields et al. (2006) found that the $\mathrm{CO}$ emission lines in a sample of $z>3$ quasars are very narrow, suggesting bulge masses about an order of magnitude lower than measured in the local Universe, at fixed BH mass (see also Coppin et al. 2008).

Lauer et al. (2007b) have discussed several possible biases which may seriously affect these findings. At high redshifts a sample will be biased toward the most luminous AGNs and more massive BHs. Given the observed scatter in the local relations, especially significant in the BHhost luminosity relation, these massive BHs will be preferentially associated with the less massive but more numerous galaxies yielding a false signal of evolution.

Several approaches have been pursued in the literature to address the issue of redshift evolution in the relations adopting methodologies which minimize the impact of observational biases. Merloni et al. (2004) compared the accreted BH mass density in AGNs with the cosmological global star formation rate density (see also Haiman et al. 2004). Although their conclusions depend on additional assumptions about the fraction of the star forming galaxies which are linked to $\mathrm{BH}$ growth at a given redshift, they were able to rule out a strong evolution at a high confidence level; we will further discuss their results below. Hopkins et al. (2006c) also found, through a model-independent integral constraint based on comparing the AGN density with the luminosity and mass functions in different bands within the redshift range $z=0 \div 2$, very significant evidence for an extremely mild evolution. 
Shankar et al. (2009a) utilized the local velocity dispersion function (VDF) of spheroids, together with their inferred age distributions, to predict the VDF at higher redshifts $(0<z<6)$. They then compared via a redshiftdependent $M_{\mathrm{bh}}-\sigma$ relation the cumulative BH mass density inferred from the AGN luminosity function with that associated with the VDF at each redshift. This comparison, insensitive to the assumed duty cycle or Eddington ratio of quasar activity, yielded a very mild redshift evolution, in agreement with previous studies.

\subsection{Other correlations between Black Holes and host galaxies}

With a more numerous sample of galaxies with updated estimates of galaxy distances, BH masses, and Sérsic indices $n$, Graham et al. (2007) found evidence for a quadratic $M_{\mathrm{bh}}-n$ relation, of the type

$$
\log \left(M_{\mathrm{bh}} / M_{\odot}\right)=(7.98 \pm 0.09)+(3.70 \pm 0.46) \log (n / 3)-(3.10 \pm 0.84)[\log (n / 3)]^{2},
$$

with an intrinsic scatter of $0.18_{-0.06}^{+0.07}$ dex and a total absolute scatter of 0.31 dex. When the quadratic relation is extrapolated, it predicts BHs with masses of $\sim 10^{3} M_{\odot}$ in $n=0.5$ dwarf elliptical galaxies, and an upper bound on the largest $\mathrm{BH}$ masses in the local Universe equal to $\sim 1.2 \times 10^{9} M_{\odot}$. This relation when adopted to compute the statistics of local BHs, yields quite different results with respect to other methods, as discussed in $\S 3$.

Marconi and Hunt (2003) explored the addition to the $M_{\mathrm{bh}}-\sigma$ relation of the parameter $r_{e}$ (the effective radius) to create a "fundamental plane" for BHs. From their 27 "Group 1" galaxies, they constructed a relation between $M_{\mathrm{bh}}$ and and $r_{e} \sigma^{2}$ (proportional to the virial bulge mass), which resulted in an intrinsic dispersion of $\sim 0.25 \mathrm{dex}$, comparable to the one in the $M_{\mathrm{bh}}-\sigma$ relation. Allowing the exponents on the $r_{e}$ and $\sigma$ terms to vary independently, Hopkins et al. (2007a) used the same 27 Group 1 galaxies from Marconi and Hunt (2003) along with some updated measurements, to find the best-fit relation

$$
\begin{aligned}
\log \left(M_{\mathrm{bh}} / M_{\odot}\right)=(8.33 \pm & 0.06)+(0.43 \pm 0.19) \log \left[r_{e} / 3 \mathrm{kpc}\right] \\
& +(3.00 \pm 0.30) \log \left[\sigma / 200 \mathrm{~km} \mathrm{~s}^{-1}\right]
\end{aligned}
$$

with an intrinsic scatter of 0.21 dex and a total scatter of 0.30 dex (see Graham 2008).

Barway and Kembhavi (2007) made the interesting claim that a combination of two photometric parameters, namely the effective radius $r_{e}$ and the 
mean effective surface brightness $\mu_{e}=-2.5 \log I_{e}$, can be used to predict BH masses with a higher degree of accuracy than single quantities such as luminosity or velocity dispersion. The optimal (B-band) solution using all 18 data points as reported by Graham (2008) is

$$
\begin{aligned}
\log \left(M_{\mathrm{bh}} / M_{\odot}\right)=(8.18 \pm 0.09)+ & (3.15 \pm 0.33) \log \left[r_{e} / 3 \mathrm{kpc}\right] \\
& -(0.900 .18)\left[\mu_{e, B}-21.0\right]
\end{aligned}
$$

with a total scatter of 0.32 dex.

The hypothesis of a BHFP which might be more fundamental than the $M_{\mathrm{bh}}-\sigma$ relation is obviously of great interest. However, later studies have questioned the actual existence of a BHFP. Graham (2008) showed that the barred early-type galaxies contained in the sample systematically deviate from the $M_{\mathrm{bh}}-\sigma$ relation, causing the residuals about the $M_{\mathrm{bh}}-\sigma$ relation to correlate with $r_{e}$ giving rise to the apparent BHFP (see, however, discussion in Younger et al. 2008). Aller and Richstone (2007) investigate a variety of correlations between bulge properties and BH mass, concluding that the strongest correlation exists between the bulge gravitational binding energy $E_{g}$, as traced by the stellar light profile, and the $\mathrm{BH}$ mass, of the type $M_{\mathrm{bh}} \propto E_{g}^{0.6}$. They, however, do not find evidence for a significant correlation between between $\mathrm{BH}$ mass, $\sigma$, and $r_{e}$ in early-type galaxies, with their best-fit relation being $M_{\mathrm{bh}} \propto \sigma^{3.73} r_{e} e^{0.05}$.

Kormendy and Bender (2009) have adopted accurate measurements of the stellar "light deficit" $L_{\text {def }}$ associated with the cores of a sample of elliptical galaxies characterized by a surface brightness covering a quite large dynamic range. They find, in agreement with several previous studies (e.g., Merritt 2006, and references therein), that $L_{\text {def }}$ correlates well with the $\mathrm{BH}$ mass, with a similar, or even smaller scatter than the one in the $M_{\mathrm{bh}}-\sigma$ relation. They claim that this might be associated to dry mergers events, during which energy can be efficiently transferred to the central stars via the anisotropic gravitational radiation emitted after the coalesce of the binary BHs (e.g., Sesana et al., 2009, and references therein). Finally, Seigar et al. (2008) claimed another correlation between the spiral arm pitch angle (a measure of the tightness of spiral structure) and the BH mass in the nuclei of disk galaxies. 


\section{The local mass function of Black Holes}

It is now widely accepted that knowing the exact number density of galaxies in all bins of stellar mass (or other property) is fundamental to discern among successful models of galaxy formation. For example (see $\S$ 8), most semianalytic models (SAMs) of galaxy formation now agree that some sort of strong stellar and/or AGN feedback must have been responsible for shaping the exponential fall-off of the stellar mass function at high stellar masses.

For similar reasons, it is essential to define the $\mathrm{BH}$ mass function (BHMF), i.e., the comoving number density of BHs per bin of mass in the local Universe. Any model of galaxy formation and evolution which attempts to provide a complete picture of galaxy evolution, must also address the problem of birth and growth of the massive $\mathrm{BHs}$ in galaxies, explain the nature of their tight relationship with their hosts, and predict their correct number and mass at $z=0$.

The standard procedure (first pioneered by Salucci et al. 1999 and Ferrarese $2002 \mathrm{~b}$ ) in calculating the BHMF assumes that all galaxies with a significant bulge component host a $\mathrm{BH}$, the mass of which can be inferred from the BH-galaxy scaling relations discussed in $\S 2$. Knowledge of a local galaxy luminosity, velocity dispersion or light concentration function, combined with the corresponding BH scaling relation, will then lead to a BHMF through a simple change of variables. The first derivations of the BHMF (e.g., Salucci et al., 1999; Ferrarese, 2002b; Aller and Richstone, 2002; McLure and Dunlop, 2004; Marconi et al., 2004; Shankar et al., 2004; Tundo et al., 2007; Graham et al., 2007) combined the galaxy velocity dispersion function (VDF) with the $M_{\mathrm{bh}}{ }^{-}$ $\sigma$ relation, and the spheroid luminosity function with the $M_{\mathrm{bh}}-M_{\text {bulge }}$ relation.

Here it is provided a brief compendium (see Shankar et al. 2009b) of the BHMFs derived from applying different $\mathrm{BH}$ mass estimators. The aim here is to provide the reader with a compact overview of the most important steps required to generate a $\mathrm{BHMF}$.

The BHMF is computed by converting the galaxy distribution $\Phi(y)$, which expresses the measured galaxy number density as a function of a given variable $y$ (e.g., the stellar velocity dispersion or bulge luminosity), into a BHMF by adopting the corresponding empirically measured $M_{\mathrm{bh}}-y$ relation. The conversion is then performed via the convolution

$$
\Phi\left(M_{\mathrm{bh}}\right)=\int \Phi(y) \frac{1}{\sqrt{2 \pi \eta^{2}}} \exp \left[-\frac{\left(M_{\mathrm{bh}}-[a+b y]\right)^{2}}{2 \eta^{2}}\right] d y
$$


which takes into account the intrinsic scatter $\eta$ in the $M_{\mathrm{bh}}-y$ relation, derived from the direct data fitting (e.g., Tremaine et al., 2002; Graham, 2007).

The BHMF can be derived by coupling the statistical information on local luminosity functions of galaxies with relationships among luminosity (or related quantities such as stellar mass and velocity dispersion) and the central BH mass (see Salucci et al. 1999). Because the BH mass correlates with the luminosity and velocity dispersion of the bulge stellar population, it is necessary to first separate the luminosity functions for different morphological types, that have different bulge-to-total luminosity ratios. It is convenient to use galaxy luminosity functions derived in bands as red as possible, where the old bulge stellar populations are more prominent.

The spheroidal luminosity function $\Phi\left(L_{\text {bulge }}\right)$ for lenticular galaxies can be computed from the luminosity function of early-type galaxies, $\Phi_{e}(L)$, as

$$
\Phi\left(L_{\text {bulge }}\right)=\frac{f_{\mathrm{S} 0}}{f_{\mathrm{E}}+f_{\mathrm{S} 0}} \Phi_{e}(b \times L),
$$

(Yu and Tremaine 2002), where $f_{E}$ and $f_{S 0}$ (which can be taken, for example, from Table 1 of Fukugita et al. 1998) are the numerical fractions of elliptical and lenticular galaxies, respectively, to the total galaxy population, and $b=L_{\text {bulge }} / L$, is the luminous bulge-to-total ratio for $S 0$ galaxies. The contribution of ellipticals to the spheroidal LF can be computed by simply setting $b=1$ and replacing $f_{\mathrm{S} 0}$ with $f_{\mathrm{E}}$ in equation (8). Similarly, one can compute the luminosity function of bulges of spirals of different Hubble type by replacing $\Phi_{e}(L)$ in equation (8) with the late-type galaxy luminosity function, and plugging in the appropriate $f$-weights for later type galaxies.

A somewhat different approach was recently adopted by Graham et al. (2007). First a BH mass via Eq. (4) and the appropriate statistical weight were associated to each spheroid in the Millennium Galaxy Catalog (Driver et al. 2007). The latter was computed as the space density of of the appropriate spheroid type in the specified color and luminosity intervals, divided by the number of galaxies which contributed to that interval. The BHMF was then computed separately for early and late-type galaxies by summing the distribution of appropriate BH masses times their weights (see Graham et al. 2007 for further details).

When computing the BHMF from the velocity dispersion function of galaxies, instead, no correction for bulges is usually applied, at least for the bulge-dominated galaxies where the velocity dispersion $\sigma$ should be a good indicator of the true $\sigma$ of the bulge component (see discussion in 
Ferrarese and Ford 2005, and references therein). Several groups therefore (e.g., Marconi et al., 2004; Shankar et al., 2004), adopted the Sheth et al. (2003) early-type velocity dispersion function, directly converted into a BHMF via the $M_{\mathrm{bh}}-\sigma$ relation. Shankar et al. (2004) also adopted the velocity dispersion function computed via the bivariate equation

$$
\Phi\left(\sigma_{i}\right)=\sum_{j} p_{i j} \Phi\left(L_{j}\right),
$$

where $p_{i j}$ is the fraction of sources in the Bernardi et al. (2003) and Sheth et al. (2003) sample with velocity dispersion $\log \sigma_{i}$ and luminosity $L_{j}$, normalized to the total number of sources with luminosity $L_{j}$, and $\Phi\left(L_{j}\right)$ is the input luminosity function. The contribution of late-type galaxies to the velocity dispersion function is more difficult to assess. Sheth et al. (2003) converted the late-type galaxy luminosity function into a velocity dispersion function by adopting a mean luminosity-velocity dispersion relation plus a model to correct for galaxy orientation. The latter result was then adopted by Shankar et al. (2009b) to compute the contribution of the bulges of spirals to the BHMF.

Figure 2 compares the local BHMFs obtained from using different relations between $\mathrm{BH}$ mass and host galaxy properties. Each line in the Figure corresponds to a different $\mathrm{BH}$ mass estimator, as listed in the legend (we defer the reader to Shankar et al. $2009 \mathrm{~b}$ for further details).

The grey band of Figure 2 can be considered as representative of the mean and the systematic uncertainties of present estimates of the BHMF. The integrated mass density of the local $\mathrm{BH}$ population is $\rho_{\mathrm{bh}}=(3.2-$ $5.5) \times 10^{5} \mathrm{M}_{\odot} \mathrm{Mpc}^{-3}$ (for $h=0.7$ ). Figure 2 also presents two additional estimates of the BHMF. The dotted curve shows the estimate, for earlytype galaxies only, by Hopkins et al. (2007a), based on the BHFP (Eq. [5]), which is in reasonable agreement with other determinations of the BHMF at the high mass end. The disagreement at the low mass end is due to the fact that the contribution from the bulges of spirals is missing from their estimate. Open circles show instead the estimate by Graham et al. (2007) based on the correlation between BH mass and the Sérsic index $n$ (Eq. 44). The latter includes a complete census of all galaxy types, and therefore the strong disagreement at the low mass end is a manifestation of a real mismatch in the results. This difference might be caused by both the use of a different relation (Eq. 4), and, as explained above, a different method with respect to that outlined in Eq. (8), although this is difficult to assess a posteriori. 


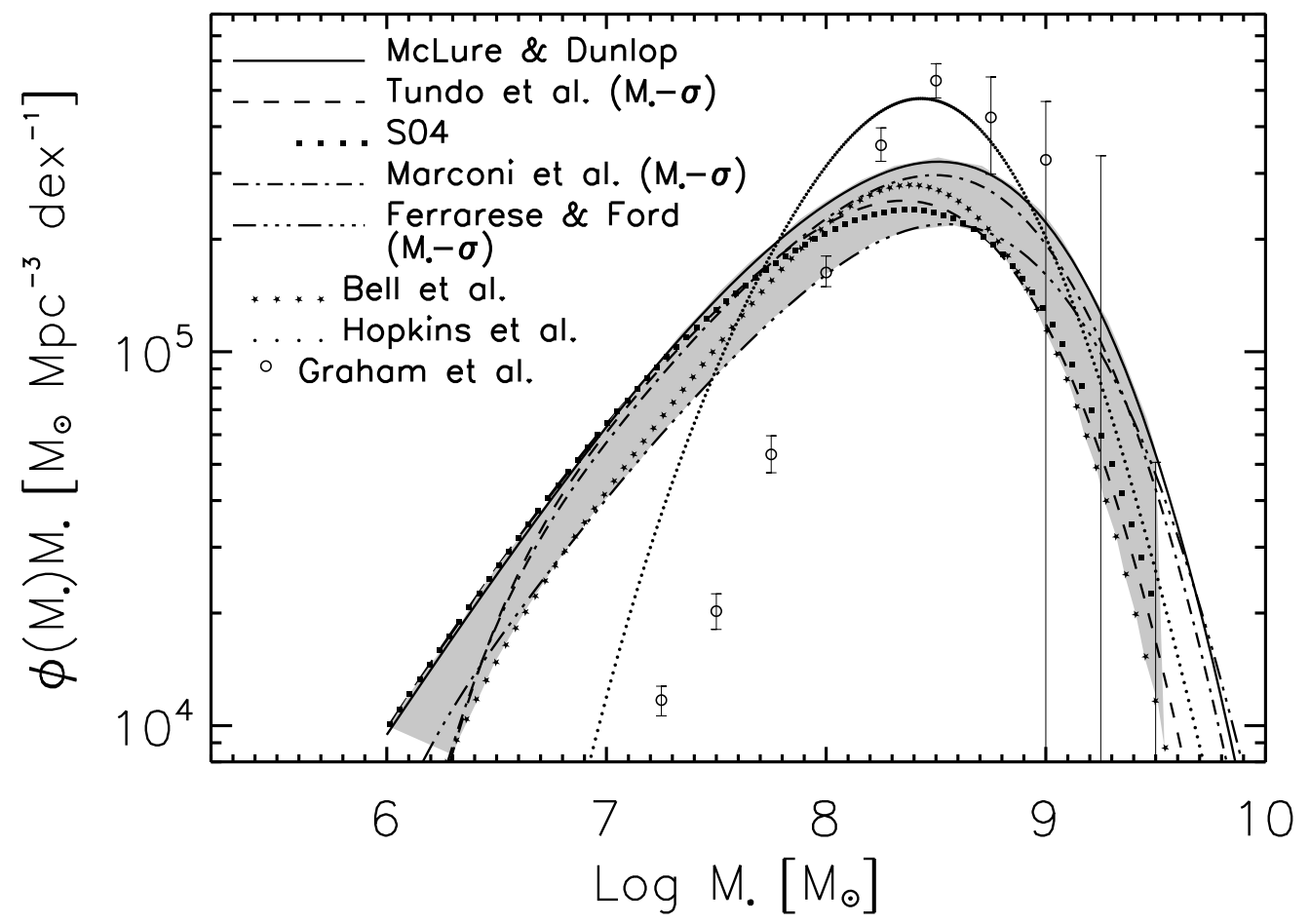

Figure 2: Comparison among estimates of the local black hole mass function taken from Shankar et al. (2009b). Lines show estimates using various calibrations of the $M_{\mathrm{bh}}-L_{\mathrm{sph}}$, $M_{\mathrm{bh}}-\sigma$, or $M_{\mathrm{bh}}-M_{\text {bulge }}$ relations, assuming a 0.3 -dex intrinsic scatter in all cases. The grey band encompasses the range of these estimates. The thick dotted line shows the determination of the early-type black hole mass function by Hopkins et al. (2007a) using the black hole "fundamental plane", and open circles show the determination of Graham et al. (2007) using the relation between black hole mass and Sérsic index. 
Nevertheless, it is interesting to note that all estimates agree in providing a similar peak in the $\mathrm{BH}$ mass distributions, which is important to constrain $\mathrm{BH}$ accretion models, as we will discuss below.

\subsection{Black Holes in Later type galaxies}

One important caveat in determining the overall census of local BHs, is the actual presence of $\mathrm{BHs}$ in bulgeless galaxies. In the previous section, the assumption has been made that all galaxies with a bulge component possess a central BH. However, there is no firm observational evidence to support this claim. On one side, bulgeless AGNs are not uncommon (e.g., Gebhardt et al., 2001; Satyapal et al., 2008), suggesting that current BHMF estimates might be missing the contribution of a $\mathrm{BH}$ population in late type spiral galaxies (although, based on current estimates, these BHs are $1-3$ orders of magnitude less massive than those discussed in this Review). On the other, the ubiquitous presence of BHs, especially in low luminosity dwarf ellipticals, has not been proven: for instance, it is questionable whether a BH exists in the E5 galaxy NGC 205 (Valluri et al. 2005). A number of recent studies, mostly with the Hubble Space Telescope, have shown that the majority of galaxies, regardless of Hubble type, host morphologically distinct stellar nuclei (e.g., Côté et al., 2006; Ferrarese et al., 2006; Boeker, 2007), the only exception (as a class) being bright galaxies with $M_{B} \lesssim-20.5$, although

cases of galaxies with both stellar nuclei and BHs do exist (e.g., Seth et al., 2008).

Evidence for BHs in smaller galaxies also come from the Chandra X-ray observations of Gallo et al. (2008), who showed that a large fraction of earlytype galaxies in the Virgo Cluster (AMUSE-Virgo) have a significant X-ray emission, consistent with what expected from accretion onto a central BH. Satyapal et al. (2008) reported the discovery of AGN activity in several local spiral galaxies, and concluded from their estimation of bolometric luminosity, that the average mass for the BHs residing in these galaxies should be around $4 \times 10^{3} M_{\odot}$, if they emit at the Eddington limit. Ghosh et al. (2008) found secure X-ray AGN detections in several nearby, face-on spiral galaxies.

It remains still open the major challenge of understanding how BHs have grown and whether they followed the assembly history of their host galaxies (see $\S$ 8). It is discussed in the following sections how, in ways independent of specific models, important constraints on how BHs have actually evolved within their host galaxies and dark matter halos can be derived by using global information on the statistics of local BHs, the statistics of active 
galaxies and their correlated clustering strengths at different luminosities and redshifts.

\section{The integrated mass density}

The classic Soltan (1982) argument relates the integrated BH density to the integrated emissivity of the AGN population, setting interesting constraints on the average radiative efficiency $\epsilon$ of BHs and its possible dependence on mass and/or redshift. If the average efficiency of converting accreted mass into bolometric luminosity is $\epsilon \equiv L / \dot{M}_{\text {inflow }} c^{2}$, where $\dot{M}_{\text {inflow }}$ is the mass accretion rate, then the actual accretion onto the central $\mathrm{BH}$ is $\dot{M}_{\text {bh }}=(1-\epsilon) \dot{M}_{\text {inflow }}$, where the factor $1-\epsilon$ accounts for the fraction of the incoming mass that is radiated away instead of being added to the BH (e.g., $\mathrm{Yu}$ and Tremaine 2002). The rate at which mass is added to the $\mathrm{BH}$ mass function is then given by

$$
\frac{d \rho_{\mathrm{bh}}}{d t}=\frac{1-\epsilon}{\epsilon c^{2}} \int_{0}^{\infty} \Phi(L) L d \log L
$$

with $\Phi(L)$ the bolometric AGN luminosity function.

Here I show a simple plot which conveys the basic idea of Soltan (1982)'s argument, while I refer to other papers for a more detailed discussion on the topic (e.g., Marconi et al., 2004; Merloni et al., 2004; Shankar et al., 2004; Tamura et al., 2006; Hopkins et al., 2007b; Merloni and Heinz, 2008; Silverman et al., 2008; Shankar et al., 2009b). Figure 3 shows the integrated accreted mass on BHs as a function of redshift following Eq. (10), for different values of the radiative efficiency $\epsilon$, as labeled. The grey stripe at $z \sim 0$ indicates the systematic uncertainties in determining the local BHMF (see Figure 2). It is clear from the Figure that the match between the local and accreted mass densities constrains the mean radiative efficiency to be $\epsilon \lesssim 0.1$. However, still significant uncertainties on the bolometric correction on one side (e.g., Elvis et al., 1994; Marconi et al., 2004; Hopkins et al., 2007b; Vasudevan and Fabian, 2007, 2009), and systematic uncertainties on the local BHMF on the other (e.g., Shankar et al., 2009b, and references therein), prevent a more precise computation of the radiative efficiency (see also discussions in Shankar et al. 2009b and Yu and Lu 2008).

Extended versions of the Soltan (1982) argument have been successfully applied to constrain other aspects of $\mathrm{BH}$ evolution. An upper limit to the degree of evolution of the $M_{\mathrm{bh}}-\sigma$ relation has been set by Shankar et al. 

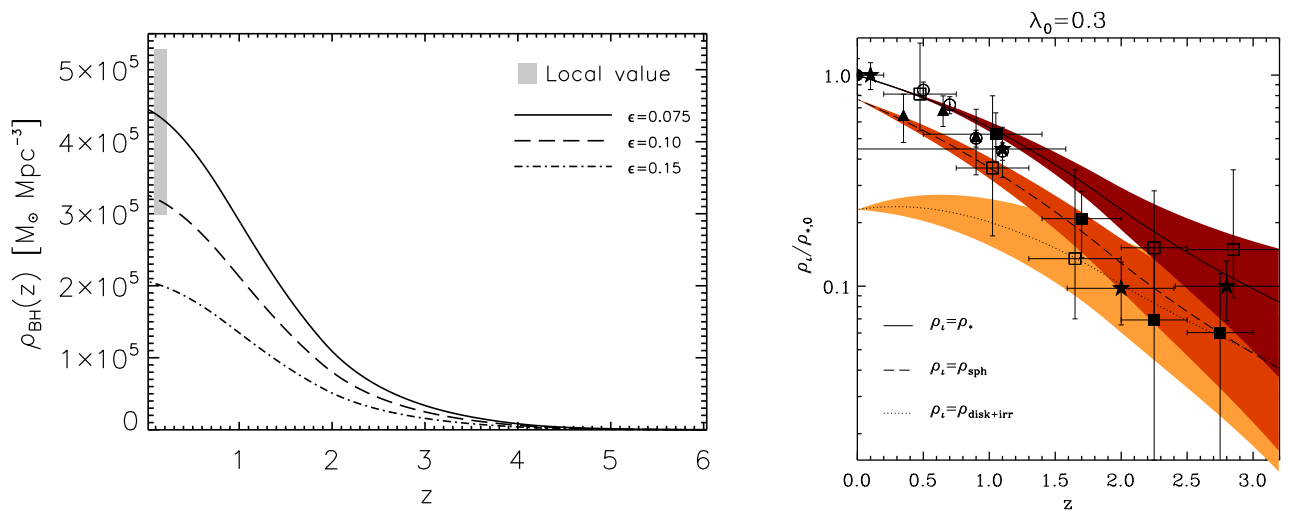

Figure 3: Left panel: integrated accreted mass on black holes as a function of redshift following Eq. (10), for different values of the radiative efficiency $\epsilon$, as labeled. The grey stripe at $z \sim 0$ indicates the systematic uncertainties in determining the local BH mass function (see Figure 2). The match between the local and accreted mass densities provides interesting constraints on the mean radiative efficiency of BHs. Right panel: evolution in the best-fitting stellar mass density as a function of redshift derived from the mean BH accretion history rescaled by a redshift-dependent constant (solid line), compared with different data sets as collected in Merloni et al. (2004). Figure taken from Merloni et al. (2004).

(2009a) by comparing the BH mass function associated with the velocity dispersion function at each redshift $z_{c}$ (corrected for galaxies with age $z<z_{c}$ ), and the accumulated total BH mass density extracted from Eq. (10); these Authors reach similar conclusions as Merloni et al. (2004), Hopkins et al. (2006c), and Shankar et al. (2009b) who used somewhat different methods. Some of these studies have also shown that the inferred average history of $\mathrm{BH}$ growth apparently parallels the cosmological star formation rate, at least in spheroids, at nearly all cosmological times, suggesting that the two processes may well be intimately linked and that, on average, BHs and galaxies may coevolve. The right panel of Figure 3 shows the result of Merloni et al. (2004). These Authors compared all the available observational data on the redshift evolution of the total stellar mass (data points in the Figure), with the integrated mass evolution of BHs, estimated from the AGN luminosity function (solid line). Assuming that the ratio of the $\mathrm{BH}$ mass density to the stellar mass in spheroids evolves as $(1+z)^{\alpha}$, they found that the match to the data requires a mild, although significant, evolution in the BH-spheroid mass ratio with $\alpha \simeq 0.5 \pm 0.2$; this implies slightly larger $\mathrm{BH}$ masses at fixed host stellar mass at $z>0$, in agreement with the evolution inferred 
by Shankar et al. (2009a) for the $M_{\mathrm{bh}}-\sigma$ relation. Also shown in the same Figure are the relative contributions from early-type (dashed line) and latetype galaxies (dotted line), with their corresponding 1- $\sigma$ confidence intervals. Merloni and Heinz (2008), Shankar et al. (2008a), and Cattaneo and Best (2009) have also used the integrated emissivity of radio sources to constrain the kinetic efficiency of BHs, in the hypothesis that radio power is correlated with the kinetic power of the jets.

\section{The evolved Black Hole mass function}

Important additional insight into the overall evolution of $\mathrm{BHs}$ can be gained by comparing not only the total mass densities, but also the shapes of the mass functions (Salucci et al. 1999). In fact, the comparison between the predicted mass distribution implied by the AGN luminosity function (Eq. [11), and the local BHMF can yield further interesting constraints on the mean Eddington ratio $\lambda$ at which $\mathrm{BHs}$ are accreting at.

The predicted mass function $n\left(M_{\mathrm{bh}}, t\right.$ ) (in units of $\mathrm{Mpc}^{-3} \mathrm{M}_{\odot}^{-1}$ ) implied by the bolometric AGN luminosity function can be derived via a continuity equation argument (e.g., Cavaliere et al., 1971; Small and Blandford, 1992; $\mathrm{Yu}$ and Tremaine, 2002; Marconi et al., 2004; $\mathrm{Yu}$ and Lu, 2004, 2008; Shankar et al., 2004; Hopkins et al., 2007b; Shankar et al., 2009b)

$$
\frac{\partial n}{\partial t}\left(M_{\mathrm{bh}}, t\right)=-\frac{\partial\left(\left\langle\dot{M}_{\mathrm{bh}}\right\rangle n\left(M_{\mathrm{bh}}, t\right)\right)}{\partial M_{\mathrm{bh}}},
$$

where

$$
\left\langle\dot{M}_{\mathrm{bh}}\right\rangle=\int S\left(M_{\mathrm{bh}}, z, \lambda\right) \lambda d \lambda M_{\mathrm{bh}} / t_{E},
$$

is the mean accretion rate (averaged over the active and inactive populations) of BHs of mass $M_{\mathrm{bh}}$ at time $t$, and $\lambda=L / L_{\mathrm{Edd}}$ the Eddington ratio, with $L_{\text {Edd }}=1.26 \times 10^{38}\left(M_{\mathrm{bh}} / M_{\odot}\right) \mathrm{erg} \mathrm{s}^{-1}$ the Eddington luminosity (i.e., $\left.\lambda \propto L / M_{\mathrm{bh}}\right)$. Eq. (11) states that the average growth rate of all BHs is proportional to the function $S\left(M_{\mathrm{bh}}, z, \lambda\right)$, i.e., the fraction of BHs of mass $M_{\mathrm{bh}}$ active at redshift $z$ and accreting at the Eddington rate $\lambda$. This evolution is equivalent to the case in which every $\mathrm{BH}$ constantly grows at the mean accretion rate $\left\langle\dot{M}_{\mathrm{bh}}\right\rangle$. In practice, individual BHs turn on and off, and there may be a dispersion in $\dot{M}_{\mathrm{bh}}$ values, but the mass function evolution depends only on the mean accretion rate as a function of mass. 
Eq. (11) can be further generalized to any distribution

$$
S\left(M_{\mathrm{bh}}, z, \lambda\right)=p(\lambda, z) \Delta\left(M_{\mathrm{bh}}, z\right) ;
$$

this assumes all active BHs at redshift $z$ to share the same mean Eddington ratio distribution $p(\lambda, z)$ (normalized to unity), and $\Delta\left(M_{\mathrm{bh}}, z\right)$ is the duty cycle, i.e., the total fraction of active BHs at redshift $z$ and mass $M_{\mathrm{bh}}$, neglecting for simplicity any further mass-dependence of the Eddington ratio distribution $p(\lambda, z)$. A physically consistent model must have $\Delta\left(M_{\mathrm{bh}}, z\right) \leq 1$ for all $M_{\mathrm{bh}}$ at all times.

In models where all BHs grow with a single value of the Eddington ratio $\bar{\lambda}, p(\lambda, z)$ is just a Dirac delta-function $p(\lambda, z)=\delta(\lambda-\bar{\lambda})$, while the duty cycle is simply the ratio of the luminosity and mass functions,

$$
\Delta\left(M_{\mathrm{bh}}, z\right)=\left.\frac{\Phi(L, z)}{\Phi_{\mathrm{bh}}\left(M_{\mathrm{bh}}, z\right)}\right|_{L \propto \bar{\lambda} M_{\mathrm{bh}}},
$$

where $\Phi_{\mathrm{bh}}\left(M_{\mathrm{bh}}, z\right)=n\left(M_{\mathrm{bh}}, z\right) M_{\mathrm{bh}} \ln (10)$, and $\Phi(L, z)$ is in units of $\mathrm{Mpc}^{-3}$ $\mathrm{dex}^{-1}$.

Once an initial condition is specified, the solution to Eq. (11) can be performed either iteratively, updating the duty cycle computed from the AGN luminosity function at the appropriate timestep (Eq. 114) or in simple integral forms, at least in the case of single- $\lambda$ models (see, e.g., $\mathrm{Yu}$ and $\mathrm{Lu}$ 2004). On adopting a broad input $p(\lambda, z)$ in which different fractions of $\mathrm{BHs}$ of the same mass are allowed to accrete at different values of the Eddington ratio $\lambda$, Eq. (11) can be efficiently solved iteratively. At any given time, given the observed AGN luminosity function $\Phi(L, z)$, the underlying BH mass function $\Phi_{\mathrm{bh}}\left(M_{\mathrm{bh}}, z\right)$, and Eddington ratio distribution $p(\lambda, z)$, the duty cycle $\Delta\left(M_{\mathrm{bh}}, z\right)$ is found fully specified by the equality (e.g., Steed and Weinberg, 2003)

$$
\Phi(L, z)=\int p(\lambda, z) \Delta\left(M_{\mathrm{bh}}, z\right) \Phi\left(M_{\mathrm{bh}}, z\right) d \log M_{\mathrm{bh}},
$$

given the input AGN luminosity function $\Phi(L, z)$ and the Eddington ratio distribution $p(\lambda, z)$.

Figure 4 shows the results from the first attempts to match the local BHMF with the BH mass function as predicted from Eq. (11) with the assumption that all BHs accrete with a single value of the Eddington ratio. The left panel shows the result worked out by Salucci et al. (1999), with the dotted and dashed lines referring to the predicted mass function for two 

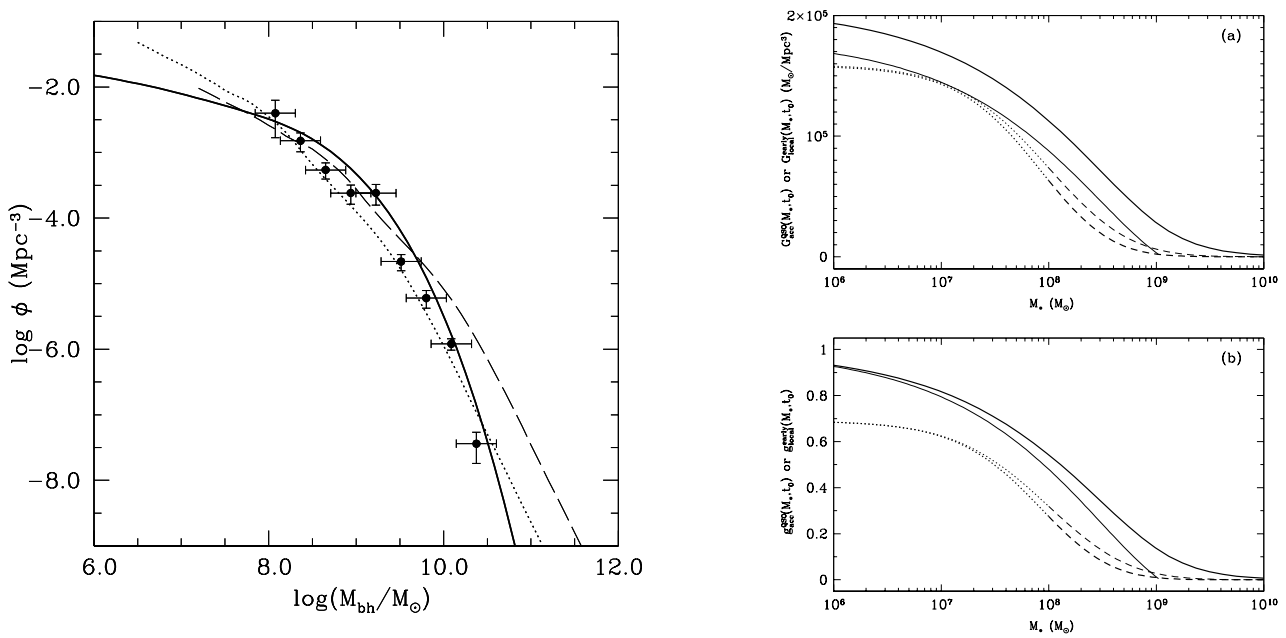

Figure 4: Left panel: predicted mass function from Eq. (11) as derived from Salucci et al. (1999) assuming $\lambda=1$ (dotted line) and $\lambda=0.2$ (dashed line). The solid line is their estimate of the local black hole mass function derived from the $M_{\mathrm{bh}}-M_{\text {star }}$ relation, while the filled symbols refer to their estimate of the local mass function derived from radio data. Figure taken from Salucci et al. (1999). Right panel: cumulative black hole mass function (thick solid line) computed from Eq. (11) by Yu and Tremaine (2002) assuming $\lambda=1$ and $\epsilon /(1-\epsilon)=0.1$ compared to local estimates of the local black hole mass function shown with dotted and dashed lines (see Yu and Tremaine 2002 for details). The thin solid line is their estimate of the predicted black hole mass function cutting out quasars radiating above a luminosity $L=L_{\mathrm{Edd}}\left(10^{9} \mathrm{M}_{\odot}\right)$. The lower panel shows the same quantities as in the upper panel normalized to unity.

values of $\lambda=1,0.2$, respectively, compared with their estimates of the local BHMF (solid line and filled symbols). The right panel shows instead a similar comparison by $\mathrm{Yu}$ and Tremaine (2002), where the solid lines show the cumulative $\mathrm{BH}$ mass functions obtained assuming $\lambda=1$ and $\epsilon /(1-\epsilon)=0.1$, compared to their estimates of the local BHMF shown with dotted and dashed lines. These preliminary works found, in agreement with the Soltan (1982) argument discussed in $\S$, that the average $\mathrm{BH}$ radiative efficiency should be around $\epsilon \lesssim 0.1$, in the assumption that most of the BH mass in the local Universe was accreted during AGN visible phases. In addition, they were the first to point out that, once $\epsilon$ is fixed using Eq. (10), the overall shape of the local BHMF can be naturally reproduced by direct integration of the AGN luminosity over time using Eq. (11), once the appropriate median $\lambda$ has been adopted. 
The upper panels of Figure 5 show the results of a reference model which evolves the predicted mass function assuming an Eddington ratio of $\lambda \sim 0.40$ and $\epsilon=0.065$ (see Shankar et al. 2009b for further details). The upper left panel of Figure 5 plots the duty cycle $\Delta\left(M_{\mathrm{bh}}, z\right)$ as a function of mass for different redshifts, as labeled. The duty cycle for $M_{\mathrm{bh}} \sim 10^{9} \mathrm{M}_{\odot}$ is $\sim 0.2$ at $z \sim 4-5$, falls to $0.03-0.08$ at $z=2-3$ when quasar activity is at its peak, then drops to 0.003 at $z=1$ and $\sim 10^{-4}$ at $z=0$. Below $z \sim 3$, the duty cycle rises towards low BH masses. This "downsizing" evolution, in which high mass BHs complete their growth early but low mass BHs continue to grow at late times, is required by the observed luminosity function evolution (e.g., Ueda et al., 2003) in any model with approximately constant $\lambda$.

This model yields overall good agreement with the average determinations of the local BHMF. The upper right panel of Figure 5 plots $M_{\mathrm{bh}} \Phi\left(M_{\mathrm{bh}}\right)$, proportional to the fraction of $\mathrm{BH}$ mass per logarithmic interval of $M_{\mathrm{bh}}$, which allows better visual comparison to the observed local BHMF and highlights the contribution of each $\mathrm{BH}$ mass bin to the total mass density at each time. The evolution of the mass function derived from the continuity equation is shown here at different redshifts from $z=3$ (dot-dashed line) down to $z=0$ (solid line). Because of the luminosity-dependent density evolution in the observed luminosity function, the massive end of the $\mathrm{BH}$ mass function builds up early, and the lower mass regime grows at later redshifts.

For $M_{\mathrm{bh}}>10^{8.5} \mathrm{M}_{\odot}$, the mass function is almost fully in place by $z=$ 1. At very high masses the model exceeds the observational estimate, but in this regime the estimate relies on extrapolation of the scaling relations, and it is sensitive to the assumed intrinsic scatter. In addition, the highmass end of the predicted mass function is sensitive to the bright end of the AGN luminosity function which might also suffer from some systematic uncertainties (see Fontanot et al. 2007, Shankar et al. 2009b and references therein). The open circles with error bars show the estimate of the local mass function by Graham et al. (2007), which cannot be reproduced even approximately with constant $\lambda$-models. If this estimate is correct, then the low end of the luminosity function must be produced mainly by high mass black holes accreting at low $\lambda$, so that the predicted growth of low mass BHs is reduced.

Assuming that all $\mathrm{BHs}$ at all times radiate at a constant Eddington ratio is obviously an oversimplification. Some empirical evidence for some sharp Eddington ratio distribution $p(\lambda, z)$ has been found by Kollmeier et al. (2006) who claim that most AGNs of different BH mass in the AGES survey at 

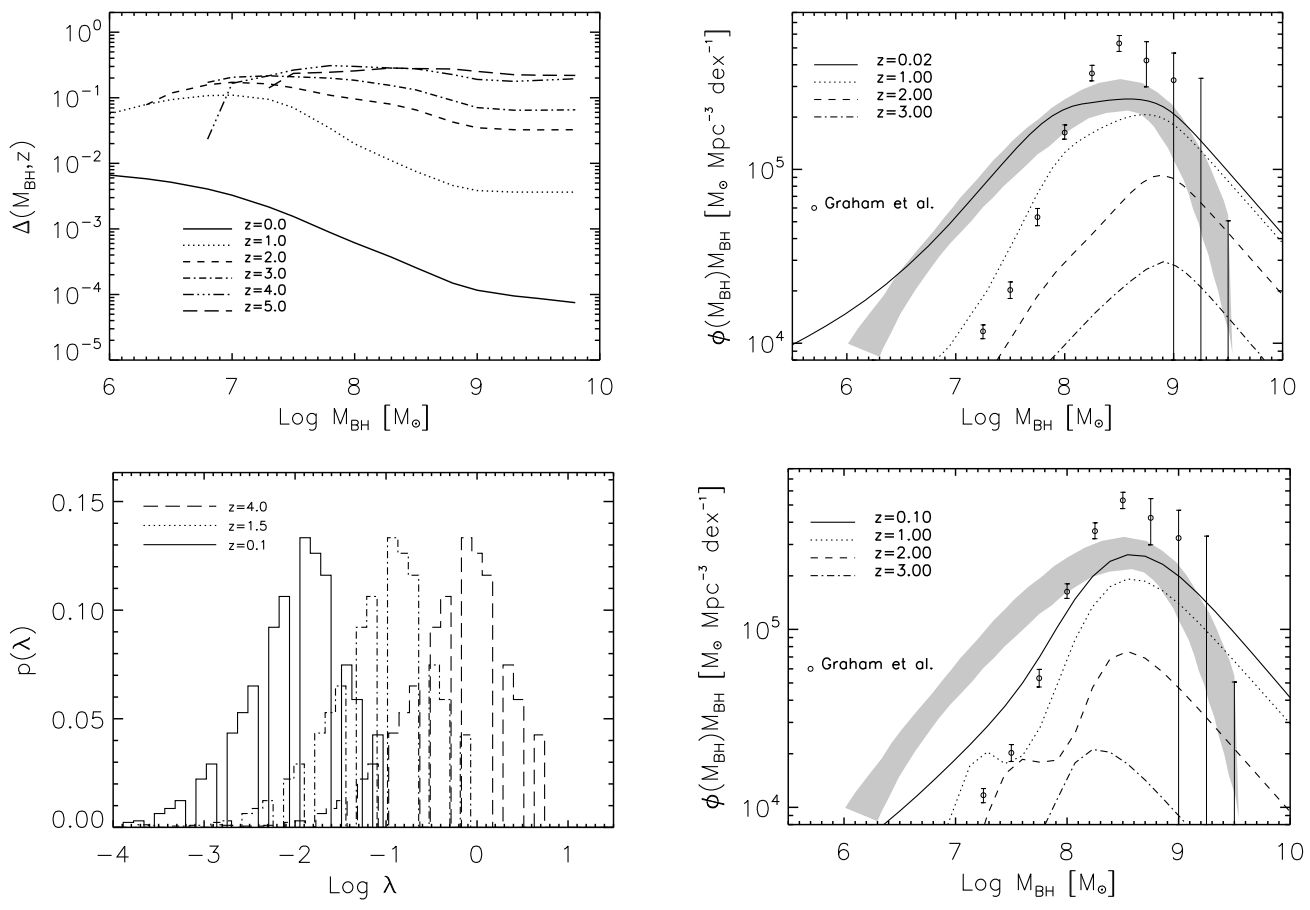

Figure 5: Lower right panel: predicted duty cycle $\Delta\left(M_{\mathrm{bh}}, z\right)$ on the basis of Eq. (11) at different redshifts and $\mathrm{BH}$ mass, as labeled. Upper right panel: predicted black hole mass function $\Phi\left(M_{\mathrm{bh}}, z\right)$ from a model with a delta-function $p(\lambda)$. The result is shown at different redshifts, from $z=3$ to $z \sim 0$, as labeled, in the plane $\Phi\left(M_{\mathrm{bh}}, z\right) M_{\mathrm{bh}}$, which plots the mass density per bin of $\mathrm{BH}$ mass. The local mass function is shown with a grey band and has a shallower slope than the one predicted by this model at $z \sim 0$ (solid line). The open circles with error bars show the estimate of the local mass function by Graham et al. (2007). Lower left panel: assumed Gaussian $p(\lambda)$ distribution with the peak steadily decreasing with decreasing redshift. Lower right panel: predicted black hole mass function $\Phi\left(M_{\mathrm{bh}}, z\right)$ from a model with a $p(\lambda)$ as given in the lower left panel. Even in this case the result is shown at different redshifts, as labeled.

$0.5 \lesssim z \lesssim 4$ all share a similar Gaussian-shaped $p(\lambda)$, constantly peaked around $\lambda \sim 0.25$ and with a dispersion of $\sim 0.3$ dex. On the other hand, at least at $z \lesssim 1$, AGN Eddington ratio distributions have a wide distribution, with a large fraction of Seyferts radiating at $\lambda<0.1$ (e.g., Hickox et al., 2009; Kauffmann and Heckman, 2008). Some studies also show evidence for strong redshift evolution and milder mass-dependence of the typical Eddington ratio (e.g., Heckman et al., 2004; Vestergaard, 2004; McLure and Dunlop, 2004; Constantin and Vogeley, 2006; Dasyra et al., 2007; Netzer and Trakhtenbrot, 

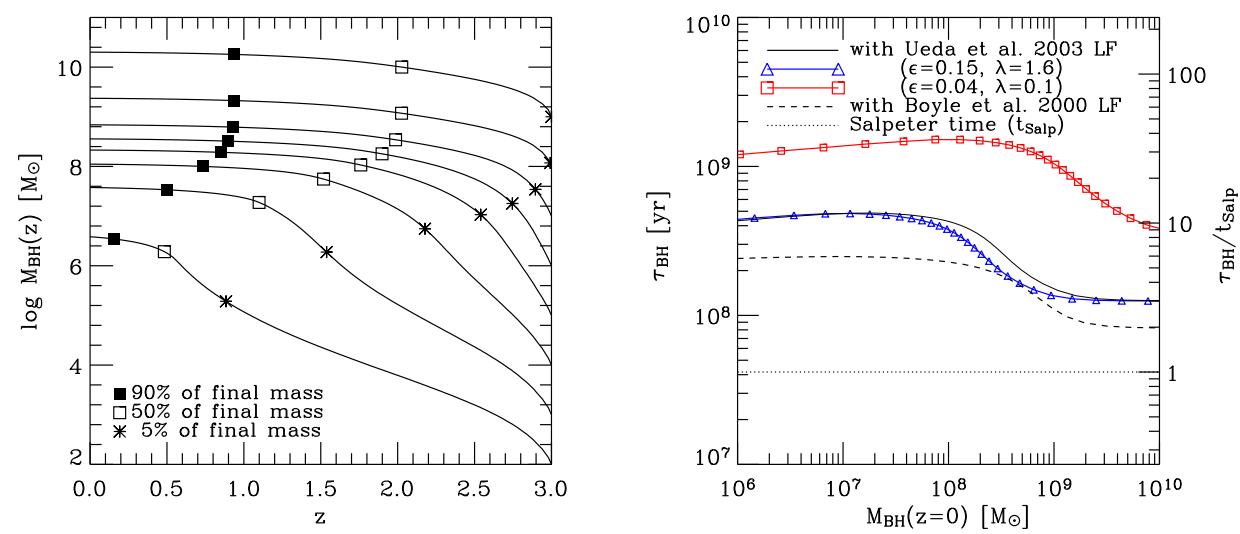

Figure 6: Left panel: average growth history of BHs with given starting mass at $z=3$ computed using the Ueda et al. (2003) luminosity function and $\epsilon=0.1, \lambda=1$. The symbols, filled squares, empty squares, and stars, indicate when the black hole reaches $90 \%, 50 \%$, and $5 \%$ of its final mass, respectively. Right panel: average mean lifetime of active BHs (AGNs) as a function of the relic BH mass at $z=0$ computed with $\epsilon=0.1$, $\lambda=1$, the Ueda et al. (2003) (solid line) and Boyle et al. (2000) (dashed line) luminosity function. The dotted line is the corresponding Salpeter time. The scale of the $y$ axis on the right is the average lifetime in units of the Salpeter time. The lines with the empty squares and triangles are the average lifetimes computed from the Ueda et al. (2003) luminosity function with $\epsilon=0.04, \lambda=0.1$, and $\epsilon=0.15, \lambda=1.6$, respectively. Figures taken from Marconi et al. (2004).

2007; Rovilos and Georgantopoulos, 2007; Shen et al., 2008), though systematic uncertainties in the reverberation mapping techniques and extrapolations of empirical virial relations (e.g., Kaspi et al., 2000; Bentz et al., 2006) make it hard to draw firm conclusions.

The lower left panel of Figure 5 shows an input $p(\lambda, z)$ with negligible mass-dependence but a strong redshift evolution (as labeled in the panel) required to be simultaneously consistent with the high- $\lambda$ observed at $z>1$ by Kollmeier et al. (2006), and the low $\lambda \sim 0.01$ observed in SDSS by, e.g., Kauffmann and Heckman (2008). At each redshift a significant spread in $p(\lambda, z)$ consistent with the observed one is also implemented. The predicted $\mathrm{BH}$ mass function $\Phi\left(M_{\mathrm{bh}}, z\right)$ implied by this model is shown in the lower right panel of Figure 5, at different redshift from $z=3$ to $z \sim 0$ as labeled, plotted in the $\Phi\left(M_{\mathrm{bh}}, z\right) M_{\mathrm{bh}}$ plane. From the comparison with the local BHMF, it is clear that the latter model still predicts a similar break for the $z=0 \mathrm{BH}$ mass function as in constant $\lambda$-models, because the average $\lambda$ is still about $0.3-0.5$. However, much less numerous low mass BHs are produced when the median 
$\lambda$ strongly decreases with redshift, because more of the low- $z$ emissivity is mapped into more massive BHs $\left(L \propto \lambda M_{\mathrm{bh}}\right)$, preventing the growth of the less massive ones. It is therefore clear that an extremely accurate estimate of the local BHMF is fundamental to discern among different $\mathrm{BH}$ accretion models (see $\S 8.2$ ). It is also possible to show that broadening the input $p(\lambda)$ distribution does not affect the downsizing of BHs at low redshifts, but produces an upsizing at higher redshifts (see also Merloni and Heinz 2008), with the fraction of more massive active BHs significantly higher than low mass ones.

Other interesting quantities which can be directly derived from the continuity equation formalism developed above are the mean growth of BHs of a given mass at $z=0$ and the average visible lifetime of BHs. The former quantity can be directly drawn from direct integration on the mean accretion rate

$$
M_{\mathrm{bh}}(z)=\int_{\infty}^{z}\left\langle\dot{M}_{\mathrm{bh}}\left(z^{\prime}\right)\right\rangle \frac{d t}{d z^{\prime}} d z^{\prime} .
$$

The result is shown in the left panel of Figure 6 as derived from Marconi et al. (2004), which shows the average growth history of BHs from $z=3$ computed using the Ueda et al. (2003) luminosity function and $\epsilon=0.1, \lambda=1$. The symbols, filled squares, empty squares, and stars, indicate when the $\mathrm{BH}$ reaches $90 \%, 50 \%$, and $5 \%$ of its final mass, respectively. Here it is apparent the notion of downsizing, where the most massive BHs acquire most of their mass at $z>1$, at variance with lower mass BHs.

The corresponding time for which a $\mathrm{BH}$ of a given mass at $z=0$ was active in the past can be derived by direct integration of the duty cycle over time, as

$$
t_{\mathrm{vis}}\left[M_{\mathrm{bh}}(z=0)\right]=\int_{\infty}^{0} \Delta\left(M_{\mathrm{bh}}, t\right) d t .
$$

The result, again taken from Marconi et al. (2004), is shown in the right panel of Figure 6 for a model with $\epsilon=0.1$ and $\lambda=1$, using both the Xray and optical luminosity functions of Ueda et al. (2003) (solid line) and Boyle et al. (2000) (dashed line), respectively. Also the outputs of other models with slightly different accretion parameters are plotted in the same Figure for comparison. It is clear that lower mass BHs have longer visibility timescales than more massive BHs. Note, however, that the latter timescale strongly depend on the epoch of "first appearance" in which the BH of that final mass starts shining in the luminosity function, and therefore cannot 
be precise estimates of $\mathrm{BH}$ lifetimes, as discussed by several Authors (e.g., Hosokawa, 2002; Yu and Tremaine, 2002; Shankar et al., 2004; Yu and Lu, 2004; Hopkins and Hernquist, 2008).

\section{Black Hole mergers}

So far we have assumed that BHs mainly grow through accretion. However, observations show, and hierarchical galaxy formation models predict, that a significant fraction of galaxies experience mergers with comparably massive galaxies during their lifetime. At least some of these galaxy mergers are likely to be accompanied by mergers of the central BHs that they contain. In fact, as the galaxies merge, the BHs should sink toward the center of the new galaxy via dynamical friction where they form a binary, which can continue its decay by transferring angular momentum to the stars intersecting its orbit. If the binarys separation decreases to the point where the emission of gravitational waves becomes efficient at carrying away the last remaining angular momentum, the BHs coalesce rapidly. However, the mechanisms that shrink the orbits of the BHs with their small cross-sections to such small scales of the order of a $\sim 1$ parsec, are not fully understood (see Merritt and Milosavljevió 2005 for a review).

In principle, including mergers in the overall evolution of the $\mathrm{BH}$ population, requires inserting a mass and redshift-dependent source term in Eq. (11). During the evolution, mergers will then redistribute mass within the $\mathrm{BH}$ population, thus varying at any given epoch the predicted mass distribution with respect to a pure accretion model. Moreover, mergers should not change the integrated mass density, so they do not affect the integrated Soltan (1982) argument discussed in the previous sections; in principle, gravitational radiation during mergers can reduce the integrated mass density, but it should not be a dominant effect (see Yu and Tremaine 2002).

However, at present it is still difficult predicting precisely the $\mathrm{BH}$ mass function implied by gas accretion and mergers. On the one side, the actual rate of $\mathrm{BH}$ merging is still highly uncertain; on the other side, the indirect important effect that mergers might have on the mass-dependence of the radiative efficiency is still unknown. In fact, there is a tight connection between radiative efficiency and angular momentum of the $\mathrm{BH}$. Most of the energy is radiated close to the innermost stable orbit, which gets closer to the central rotation axis if the $\mathrm{BH}$ is rapidly spinning (see, e.g., Bardeen et al. 1972 for the formalism). Therefore a high spin is paralleled by a high radiative 
efficiency. BHs are believed to spin up or spin down after mergers and/or gas accretion, although the details of such processes are too poorly understood to allow detailed modeling. The canonical value of the radiative efficiency competing to a non-rotating $\mathrm{BH}$ is $\epsilon \sim 0.06$, increasing up to $\epsilon \sim 30 \%$ for a rapidly spinning $\mathrm{BH}$ in equilibrium (Thorne, 1974). The value of the radiative efficiency $\epsilon \lesssim 0.1$ inferred from the Soltan (1982) argument discussed in $\S 4$ (see Figure 3), appear to indicate that on average BHs are not extreme rotators, although the caveats discussed in $\S 4$ might bias this conclusion.

Hughes and Blandford (2003) studied the remnants of merging BHs of different sizes, spins and orbital parameters. They concluded that BHs are generally spun down by mergers and only for a very narrow range of orbital parameters merging is effective in rapidly spinning up the holes. Gammie et al. (2004) carried out numerical simulations confirming the Hughes and Blandford (2003) result that a $\mathrm{BH}$ is usually spun down by frequent minor mergers, while major mergers usually spin up the BHs.

Volonteri et al. (2005) studied the expected distribution of $\mathrm{BH}$ spins and its evolution with cosmic time in the context of hierarchical galaxy formation theories. They followed the merger history of dark matter halos as extracted from Monte Carlo realizations based on the Extended Press-Schechter formalism, and followed the growth of BHs via gas accretion and mergers. They concluded that mergers do not lead to a systematic spin up or spin down of BHs with time, but actually the spin distribution retains memory of its initial conditions. In their models, accretion is the main driver for efficiently spinning BHs up, and they find that if accretion takes place from a thin disk, then up to $70 \%$ of all $\mathrm{BHs}$ can be maximally rotating and have radiative efficiencies $\gtrsim 12 \%$. The Volonteri et al. (2005) conclusion was based on the assumption that the $\mathrm{BH}$ spin aligns very rapidly with the accretion flow, although they also note that a randomly-oriented sequence of accretion events is less efficient in spinning the BH up. King and Pringle (2006), strengthen the latter point, discussing through analytical methods how a randomly oriented sequence of accretion episodes can effectively keep the spin low, rather than aligning the hole very quickly and causing systematic spin up. Berti and Volonteri (2008) used results from numerical relativity simulations of $\mathrm{BH}$ mergers, coupled to the cosmological evolution of $\mathrm{BH}$ spin, to infer the evolution of the spin distribution. They conclude that mergers are very unlikely to yield substantial spins, unless alignment of the spins of the merging holes with the orbital angular momentum is very effective.

Shankar et al. (2009b) have illustrated the potential impact of mergers 
on the predicted BH mass function using a simple, yet useful mathematical model that assumes constant probability of equal mass mergers per Hubble time, similar to the models of Steed and Weinberg (2003). Following the latest results discussed above on the relatively mild effect of mergers on the spin distribution, Shankar et al. (2009b) neglected the variations of radiative efficiency caused by the impact of mergers on the BH spin distribution, and also secondary effects such as ejection of BHs by gravitational radiation or threebody interactions (e.g., Hughes and Blandford, 2003; Gammie et al., 2004; Islam et al., 2004; Yoo and Miralda-Escudé, 2004; Volonteri et al., 2005; Merritt and Milosavljević, 2005; Yoo et al., 2007; Sesana et al., 2009).

Shankar et al. (2009b) assumed that a BH of mass $M_{\mathrm{bh}}$ has a probability $P_{\text {merg }}$ of merging with an equal mass black hole in the Hubble time $t_{H}(z)$ (age of the Universe at redshift $z$ ). Therefore the fraction $F$ of black holes that merge in a timestep $\Delta t$ is given by

$$
F=P_{\operatorname{merg}} \times \frac{\Delta t}{t_{H}(z)} .
$$

At each time $t_{1}$, these Authors first advance the mass function to time $t_{2}=$ $t_{1}+\Delta t$ with accretion only, then add to each bin of the mass function an increment (which may be positive or negative)

$$
\Delta \Phi\left(M_{\mathrm{bh}}, t_{2}\right)=\frac{F \times \Phi\left(\frac{M_{\mathrm{bh}}}{2}, t_{2}\right)}{2}-F \times \Phi\left(M_{\mathrm{bh}}, t_{2}\right),
$$

where the second term represents BHs lost from the bin by merging and $\Phi\left(\frac{M_{\mathrm{bh}}}{2}, t_{2}\right)$ is calculated by interpolation.

Figure 7 shows the evolution of the $\mathrm{BH}$ mass function resulting from a model with $P_{\text {merg }}=0.5, \epsilon=0.065$ and $\lambda \sim 0.4$. The net effect of merging is to slightly lower the abundance of BHs below the peak of the mass function and to significantly increase the abundance of very massive BHs, as expected (see also Malbon et al. 2007, Yoo et al. 2007). Since a non-merger model already produces an excess of massive BHs relative to the local BHMF (see Figure 5), adding mergers only makes the match to observations worse. However, the impact of mergers is evident mainly for $M_{\mathrm{bh}}>10^{9} \mathrm{M}_{\odot}$, where the BHMF estimates are most sensitive to the adopted scatter in the black hole-host scaling relations and to the extrapolations of these relations up to the most luminous galaxies. Note that the value $P_{\text {merg }}=0.5$ is an upper limit for the merger rate of massive galaxies (see, e.g., Bundy et al. 2009 and references 


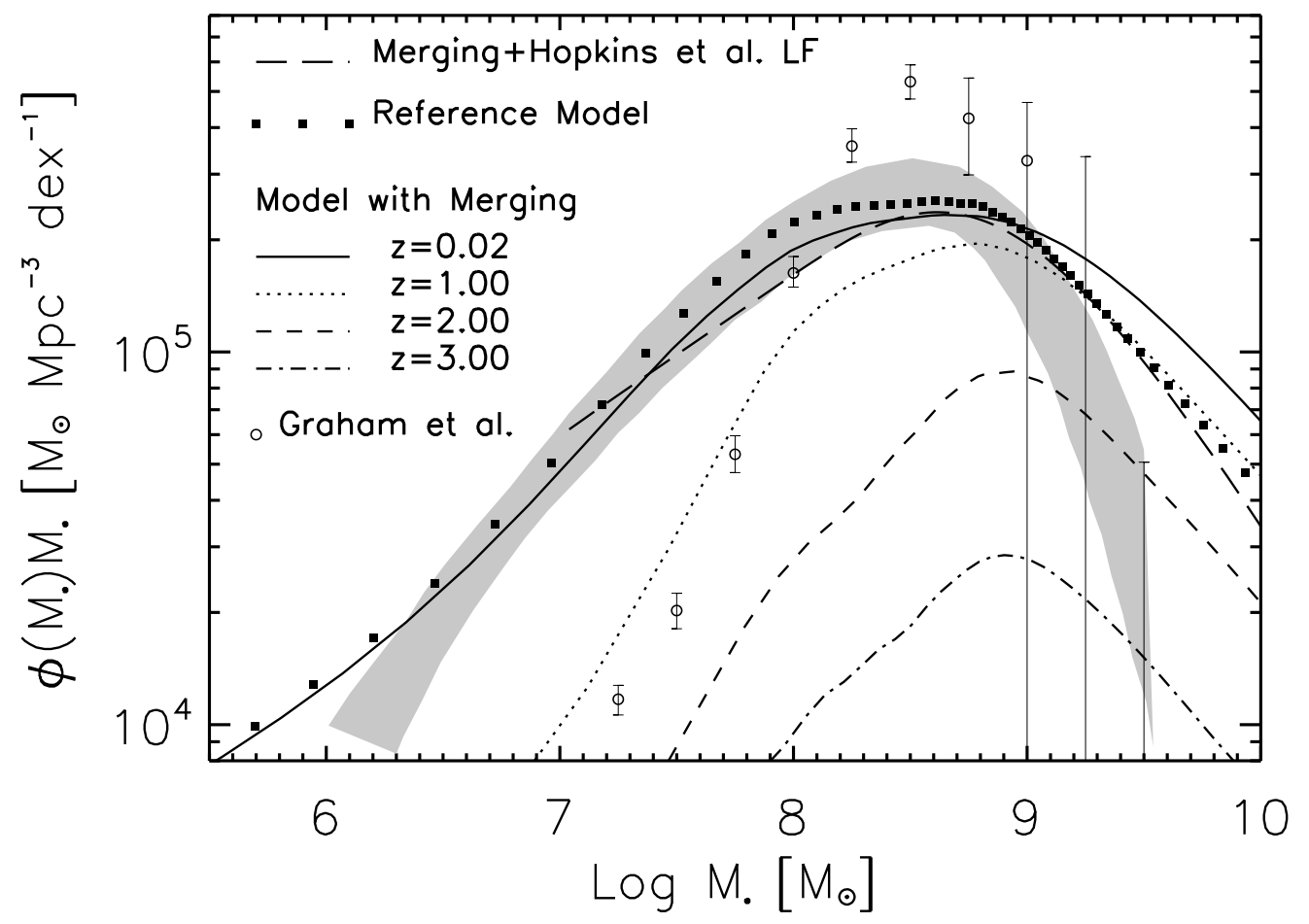

Figure 7: Evolution of the black hole mass function in a model with black hole mergers. Accretion-driven growth is computed assuming $\epsilon=0.065, \lambda=0.40$, and each black hole has a probability $P_{\text {merg }}=0.5$ of merging with another black hole of equal mass per Hubble time $t_{H}(z)$. Squares show the $z=0$ predictions of the reference model (same accretion parameters, no merging), and the long-dashed line shows the $z=0$ mass function for a merger model with the Hopkins et al. (2007b) luminosity function and accretion parameters $\epsilon=0.09, \lambda \sim 1$. 
therein), and it was chosen to provide an upper limit on the actual impact of mergers on the $\mathrm{BH}$ population.

The impact of mergers on the predicted $\mathrm{BH}$ mass function is likely to be small compared to remaining uncertainties in accretion-driven growth, except perhaps for the rare, high mass BHs. The most interesting impact of BH mergers may arise indirectly, through their effect on $\mathrm{BH}$ spins and thus on radiative efficiencies, as discussed above (e.g., Volonteri et al., 2005). Assuming, for example, the radiative efficiency to increase with increasing $\mathrm{BH}$ mass, might be a viable solution to accommodate a sequence of numerous mergers with the extremely steep fall-off of the local BHMF at the high-mass end (see also Cao and Li 2008). Potential evidences for some mass and/or redshift dependence of the radiative efficiency may already be available (see $\S$ (7).

Figure 8 illustrates my summary of the main constraints which can be derived by comparing the predictions of accretion plus mergers models for $\mathrm{BH}$ evolution with the local BHMF. The overall BH mass density constrains the radiative efficiency as first pointed out by Soltan (1982), while the peak of the mass distribution is able to constrain the mean Eddington ratio (Salucci et al. 1999). Matching the low end of the mass distributions can instead shed light on the alternative between models with low-mass BHs growing at a significant fraction of the Eddington limit, and models marked by higher-mass BHs growing at sub-Eddington regimes (see $\S$ - ). Finally, the high-mass end, coupled with some information on the mass-dependence of the radiative efficiency, should provide useful constraints on the impact of mergers on the overall $\mathrm{BH}$ evolution.

\section{AGN CLUSTERING PROVIDES INDEPENDENT CONSTRAINTS ON BLACK HOLE EVOLUTION}

In the previous sections it has been shown that continuity equation arguments based on evolving the $\mathrm{BH}$ population linking $\mathrm{BH}$ growth to the observed AGN bolometric luminosity function yields interesting constraints when compared to the local BHMF. However, this technique can only constrain mean quantities. For example, it cannot distinguish between a model with a constant $\lambda=0.4$ at all redshifts, or a model with Super-Eddington accretion $(\lambda>1)$ at higher redshifts, steadily decreasing to sub-Eddington regimes at lower- $z$ (lower panels of Figure 5). On the other hand, there are no assumption-free direct observations of the radiative efficiency at high red- 


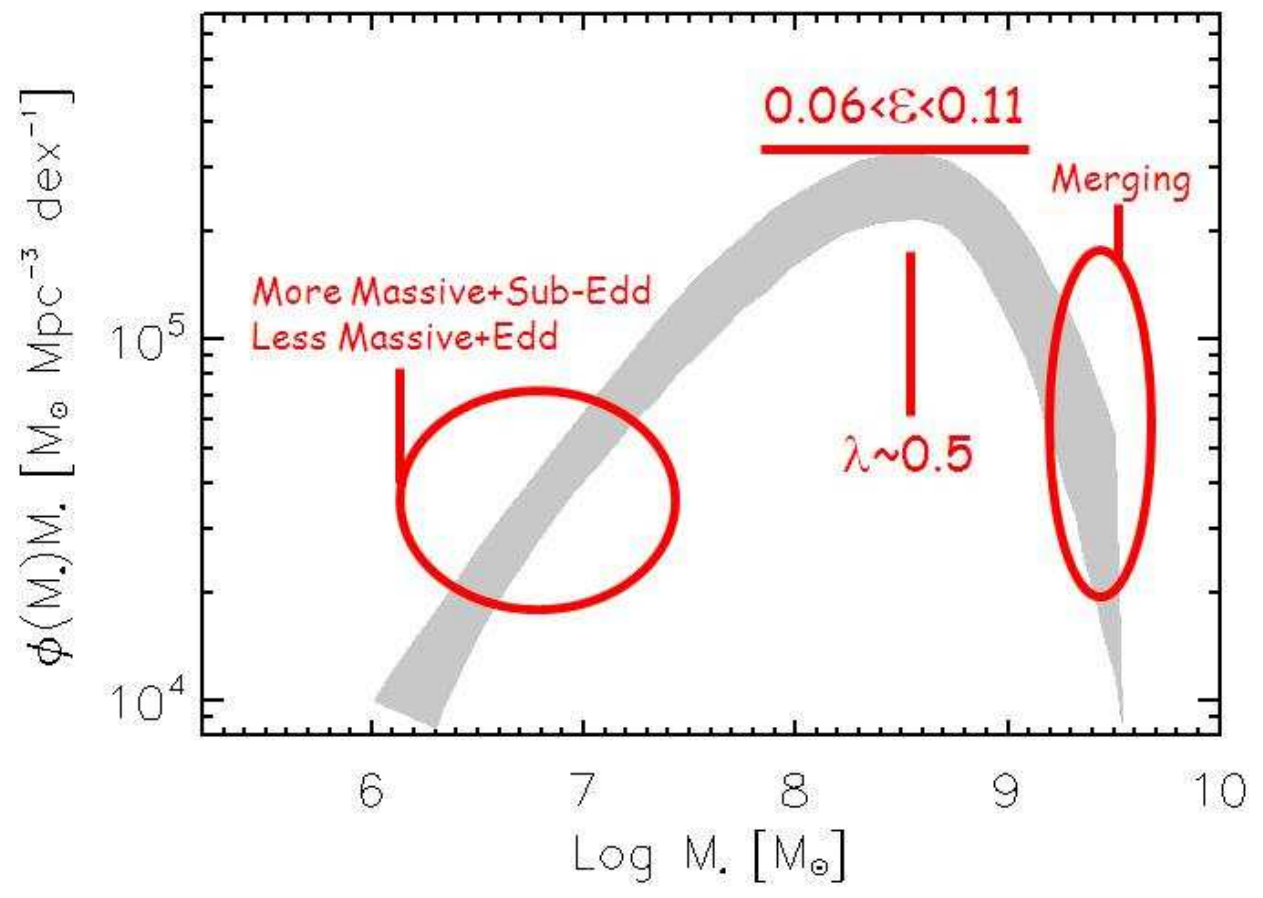

Figure 8: Summary of the main constraints which can be derived by comparing the predictions of accretion plus mergers models for black hole evolution with the local black hole mass function (grey area). The overall height under the curve constrains the radiative efficiency, while the peak of the mass distribution is able to constrain the mean Eddington ratio. Matching the low end of the mass distributions can instead shed light on the alternative between models with low-mass BHs growing at a significant fraction of the Eddington limit, and models marked by higher-mass BHs growing at sub-Eddington regimes. The high-mass end, coupled with some information on the mass-dependence of the radiative efficiency, should instead provide useful constraints on the impact of mergers on the overall $\mathrm{BH}$ evolution. 
shifts (e.g., Rafiee and Hall, 2009; Wang et al., 2009, and references therein), and a constant as well as a redshift-dependent radiative efficiency can still satisfy the Soltan (1982) argument. Breaking such degeneracies would require knowledge of the $\mathrm{BH}$ mass function at higher redshifts, which is still not feasible given that it is still not clear whether the local relations between BHs and galaxies still hold at higher redshifts (e.g., Shankar et al., 2009a, and references therein).

Quasar clustering provides additional, independent constraints on the main parameters regulating BH evolution (e.g., Mo and White, 1996; Haehnelt et al., 1998) and their possible evolution with redshift and/or mass, thus helping in discriminating among the successful models. As first outlined by Martini and Weinberg (2001) and Haiman \& Hui (2001; see also Lidz et al. 2006; Hopkins et al. 2008; Shankar et al. 2008b; White et al. 2008; Bonoli et al. 2008; Marulli et al. 2009; Thacker et al. 2009), the clustering is in fact a direct measure of the masses, and therefore number densities, of the halos hosting the quasars. In turn, the ratio between the quasar luminosity function and the halo mass function provides information on the duty cycle, i.e., the fraction of halos which shine as quasars at a given luminosity, $\mathrm{BH}$ mass, or Eddington ratio.

Many groups have now been able to measure the clustering of AGNs at different luminosities, bands, scales, and redshifts (e.g., Overzier et al., 2003; Grazian et al., 2004; Croom et al., 2005; Constantin and Vogelev, 2006; Hennawi et al., 2006; Porciani et al., 2004; Porciani and Norberg, 2006; da Ângela et al., 2008; Mvers et al., 2007a.b; Padmanabhan et al.,, 2008; Shen et al., 2007, 2009; Coil et al., 2007, 2009; Miyaji et al., 2007; Hickox et al., 2009; Ross et al., 2009, and references therein). AGN clustering is usually expressed in terms of the bias, i.e., the square root of the ratio between the matter and the measured two-point correlation functions. It is beyond the scope of this Review to discuss and compare all the above mentioned empirical results. In the following we will just focus on the semi-empirical methods which have been adopted in the literature to actually use such measurements for deriving firm constraints on $\mathrm{BH}$ evolution.

The classical modeling of quasar clustering by Haiman \& Hui (2001) and Martini \& Weinberg (2001) assumes a mean value for the duty cycle and derives the relation between quasar luminosity and host halo mass by monotonically matching their cumulative distribution functions. Formally, 
this concept leads to

$$
\begin{array}{r}
\int_{x_{\min }}^{\infty} \Phi(x, z) d x=\int_{0}^{\infty} d y \Delta_{h}(y, z) \Phi_{h}(y, z) \times \\
\frac{1}{2} \operatorname{erfc}\left[\ln \left(\frac{10^{y_{\min }\left(x_{\min }\right)}}{10^{y}}\right) \frac{1}{\sqrt{2} \ln (10) \Sigma}\right]
\end{array}
$$

here $x=\log L$ ( $L$ being the quasar luminosity), and $y=\log M_{h}$ ( $M_{h}$ being the halo mass). The quantity $\Phi_{h}(y, z)$ is the comoving number density of halos, in units of $\mathrm{Mpc}^{-3} \mathrm{dex}^{-1}$ (e.g., Sheth et al., 2001), while $\Phi(x, z)$ is the luminosity function of quasars in the same units of $\mathrm{Mpc}^{-3} \mathrm{dex}^{-1}$. The quantity $\Delta_{h}(y, z)$ in Eq. (20) is the duty cycle, i.e., the fraction of halos which host quasars shining above a minimum luminosity $x_{\min }=\log L_{\min }$ at redshift z. Eq. (20) also takes into account a scatter of $\Sigma$ (in dex) between quasar luminosity and halo mass.

So the clustering clearly provides an independent way to constrain duty cycle $\Delta_{h}(y, z)$ of active halos and BHs, which can be directly compared with the $\Delta\left(M_{\mathrm{bh}}, z\right)$ predicted from continuity equation arguments (see Eq. [14]). Either method can be adopted independently to work simultaneous constraints on the radiative efficiency, Eddington ratio distributions, and the underlying $\mathrm{BH}$ mass distribution at a given redshift; this ultimately provides in ways independent of specific models, powerful tools to delineate a comprehensive picture of $\mathrm{BH}$ evolution. Also, these determinations of the duty cycles can be further constrained by comparing with direct estimates of the fraction of active galaxies in complete samples above a certain luminosity (e.g., Kauffmann et al., 2003).

At each redshift Eq. (20) defines the minimum halo mass $y_{\text {min }}$ corresponding to the minimum luminosity in the sample $x_{\min }$. The mean bias associated to the same subsample at redshift $z$ is then

$$
\langle b\rangle=\frac{\int_{y_{\min }}^{\infty} d y \Phi_{h}(y, z) b(y, z)}{\int_{y_{\min }}^{\infty} d y \Phi_{h}(y, z)} .
$$

Martini and Weinberg (2001) and Haiman and Hui (2001) applied Eq. (20) showing that quasar clustering measurements can substantially narrow down the range of possible duty cycles. In fact, if quasars are long-lived they are rare phenomena that are highly biased with respect to the underlying dark matter; whilst if short lived, they reside in more typical halos that are less 

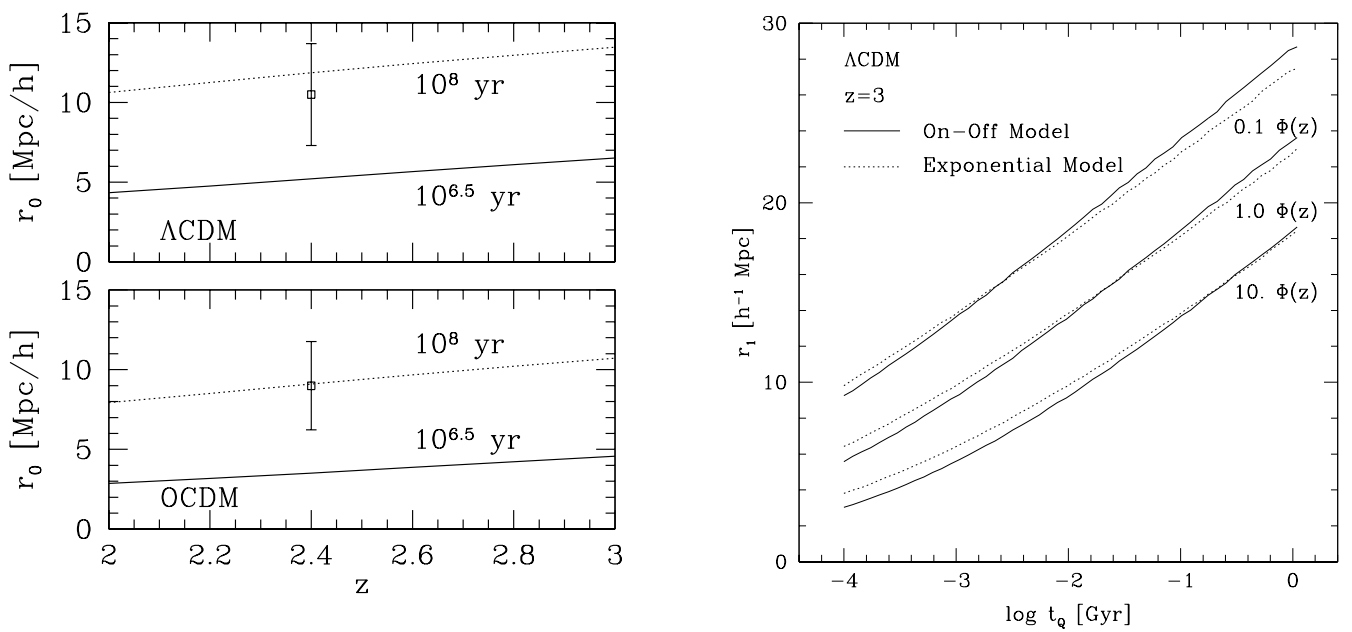

Figure 9: Left panel: correlation length predicted for two different quasar lifetimes, as labeled. Figure taken from Haiman and Hui (2001). Right panel: clustering length vs quasar lifetime $t_{Q}$ and for different values of the quasar number density $\Phi$, as labeled. Figure taken from Martini and Weinberg (2001). The main point in both panels is that an higher quasar lifetime (i.e., an higher duty cycle) implies the quasars to be hosted in less numerous, more massive and more biased halos.

strongly clustered. Figure 9 shows examples of their calculations in which the duty cycles are actually expressed in terms of the quasar optical lifetime $t_{Q}$, which is directly proportional to the duty cycle $\Delta_{h}$, for given approximations as to the lifetime of the host halos. Both plots show that the clustering strength increases for increasing quasar lifetime $t_{Q}$ and, at fixed $t_{Q}$, increases for decreasing quasar number density $\Phi$, as labeled. This behavior is easily understood given that longer quasar lifetimes (i.e., an higher duty cycle), and/or lower quasar abundances, imply quasars to be hosted in less numerous, more massive, and more biased halos.

Eq. (21) can be generalized to compute the bias as a function of quasar luminosity. Assuming that quasar luminosity closely tracks halo mass, one would naively expect an increasing clustering strength for more luminous quasars. While this seems to indeed be the case for high-redshift quasars Francke et al. (2008); Shen et al. (2009), lower redshift surveys find evidence instead for a much flatter quasar bias as a function of luminosity. Lidz et al. (2006) investigated the luminosity dependence of quasar clustering in more detail and concluded that by adopting more complicated, non-monotonic quasar light curves, such as the ones predicted from numerical simulations 
Hopkins et al. (2006a), a better match to the low- $z$ data can be found. This is because in this picture the Eddington ratio distribution at fixed $\mathrm{BH}$ mass is much broader, thus mapping both fainter and brighter quasars into similar bins of $\mathrm{BH} /$ halo mass, and therefore flattening the bias as a function of luminosity. The result of their model is shown in Figure 10 (dotted and solid lines), compared to a model (dashed line) which maps quasars into halos with a nearly one-to-one relation, and thus predicts a much stronger rise of the bias with increasing luminosity.

Other important constraints can also be derived by studying the scaledependence of the quasar bias. If luminous quasars are predominantly triggered by merging/interaction events, then their clustering strength at small scales should be enhanced with respect to a random non merger population with similar masses and large-scale clustering properties. Indeed, such a small-scale excess has been seen, in both low-redshift Serber et al. (2006) and high-redshift Hennawi et al. (2006); Mvers et al. (2007b) quasar populations. Interestingly, however, Serber et al. (2006) see no such excess in the low- redshift Seyfert population, as shown in Figure 11, which plots the excess small-scale clustering of quasars and Seyferts with data taken from Serber et al. (2006) for optical quasars (red filled squares) and Seyferts (black open squares). The lines show the predicted predicted bias as a function of scale radius determined from the correlation function at each radius relative to that at large scales, as predicted from the evolutionary models of Hopkins and Hernquist (2009). Interestingly, Li et al. (2008) using SDSS data showed that while specific star formation rates of galaxies are higher if they have close neighbors, close neighbors are not associated with any similar enhancement of nuclear activity. This leads to conclude that star formation induced by a close companion and star formation associated with $\mathrm{BH}$ accretion are distinct events. They also suggest that these events might still be part of the same physical process, for example a merger, provided they are separated in time. These empirical results, as well as those by Serber et al. (2006), support a scenario in which lower luminosity, often lower mass BHs, as usually associated with Seyfert galaxies, might be more likely triggered by in-situ phenomena, at variance with the violent dynamical events which are believed to be at the origin of the high- $z$, luminous quasars (e.g., Sanders et al. 1988, see also Kawakatu et al. 2003 and $\S$ 8).

Particularly interesting constraints can be derived from the recent $z>3$ clustering measurements of luminous quasars in SDSS by Shen et al. (2007). White et al. (2008) have applied Eqs. (201) and (21) to the data, concluding 


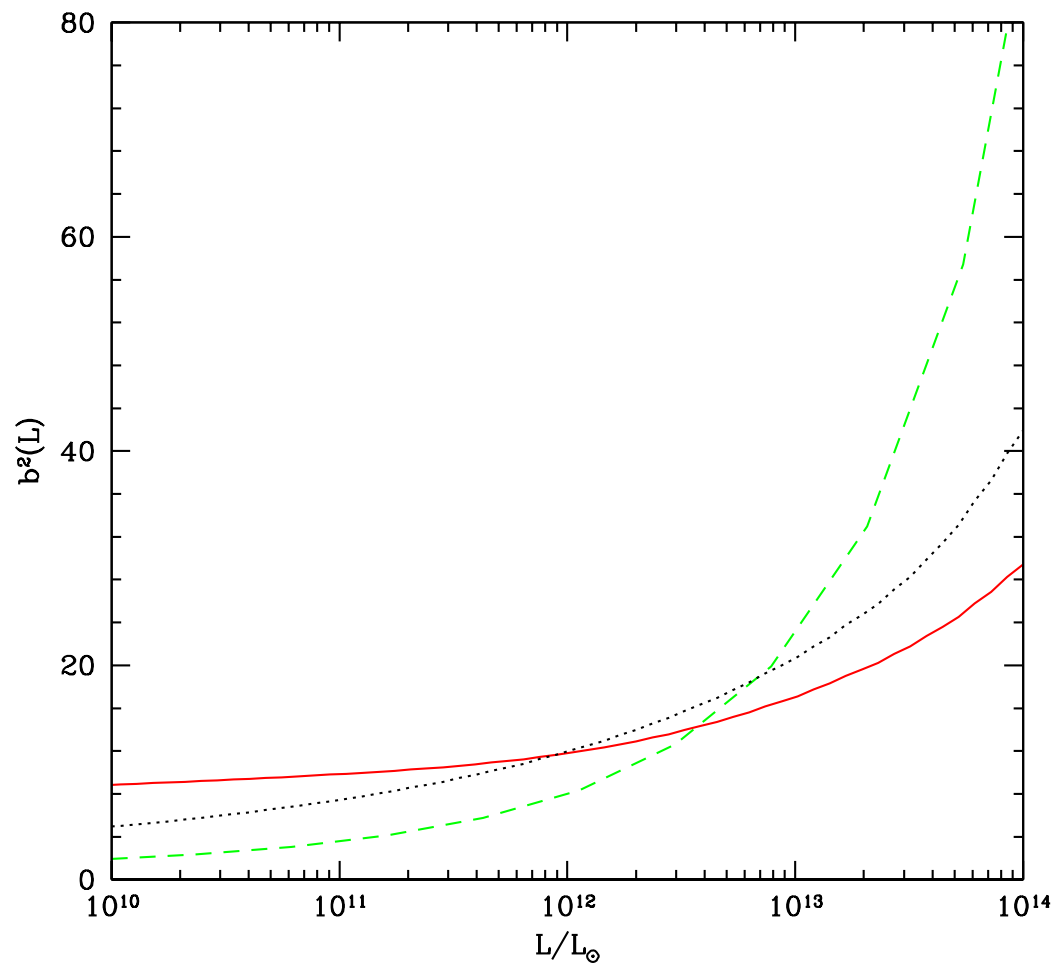

Figure 10: Bias-squared of quasars as a function of their luminosity. The solid and dotted lines show the luminosity dependence of quasar clustering in a model with a non-linear light curve as predicted from numerical simulations, while the dashed line refers to a strictly monotonic model between quasar luminosity and halo mass. Figure taken from Lidz et al. (2006). 


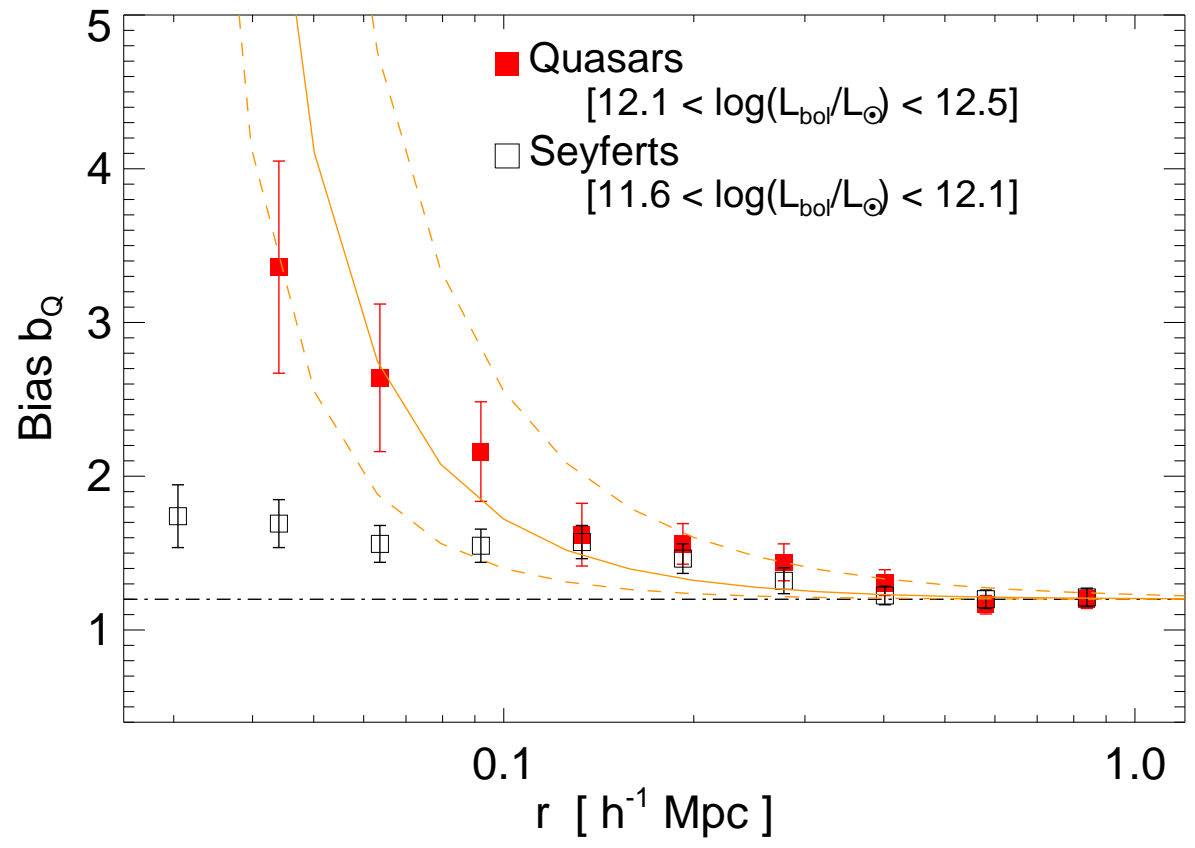

Figure 11: Excess small-scale clustering of quasars and Seyferts. The data are from Serber et al. (2006) for optical quasars (red filled squares) and Seyferts (black open squares). Plotted is the predicted bias as a function of scale radius determined from the correlation function at each radius relative to that at large scales (i.e., dividing out the best-fit large-scale dark matter correlation function). The dotteddashed line shows a constant bias as a function of scale (i.e., no excess of small-scale densities, expected if the galaxies are a random subpopulation with no preference for small groups), the solid (dashed) orange line shows the predicted excess on small scales expected for recent merger populations from cosmological models and simulations (reflecting a preference for small group environments). Figure taken from Hopkins and Hernquist (2009). 
that the strong clustering measured at $z \sim 4$ can be understood only if quasar duty cycles are high and the intrinsic scatter in the luminosity-halo relation is small. Shankar et al. (2008b) took a further step by jointly considering the evolution of the $\mathrm{BH}-$ halo relation and the $\mathrm{BH}$ mass function, as constrained by the observed AGN luminosity function and clustering. They examined constraints on the host halos, duty cycles, radiative efficiencies, and mean Eddington ratios of massive $\mathrm{BHs}$ at $z>3$, as imposed by the clustering measurements of Shen et al. (2007) and by a variety of measurements of the quasar luminosity function at $3 \leq z \leq 6$ (e.g., Hopkins et al., 2007b; Shankar and Mathur, 2007; Fontanot et al., 2007; Shankar et al., 2009b, and references therein). They linked $\mathrm{BH}$ growth to halo growth via a relation between $\mathrm{BH}$ mass and halo virial velocity $V_{\text {vir }}$. They found that the strong clustering of AGNs observed at $z=3$ and, especially, at $z=4$ implies that massive BHs reside in rare, massive dark matter halos. In turn, reproducing the observed luminosity function as implied by the growth of dark matter halos, requires high efficiency $\epsilon$ and/or low Eddington ratio $\lambda$, with a lower limit (based on $2 \sigma$ agreement with the measured $z=4$ correlation length) $\epsilon \gtrsim 0.7 \lambda /(1+0.7 \lambda)$, implying $\epsilon \gtrsim 0.17$ for $\lambda>0.25$. The rapid drop in the abundance of the massive and rare host halos at $z>7$ also implies a proportionally rapid decline in the number density of luminous quasars, much stronger than simple extrapolations of the $z=3-6$ luminosity function would predict.

Figure 12 shows a compact summary of their main result. The left panel shows that only assuming maximal values of the duty cycle $\Delta$ the predicted clustering strength (solid and dashed lines) can be, at least marginally, consistent with the data at $z=4$ (grey bands). The right panel shows with horizontal stripes the integrated BH mass density above $L=10^{45} \mathrm{erg} \mathrm{s}^{-1}$ and $4.0<z<6$ derived from the AGN luminosity function for three different values of the radiative efficiency, as labeled. The triple dot-dashed, solid, long-dashed, and dot-dashed lines indicate the BH mass density implied by the AGN luminosity function at $z=4.0$ integrated over mass assuming a mean Eddington ratio $\lambda=0.1,0.25,0.5,1$, respectively, as a function of duty cycle $\Delta$. High duty cycles imply quasars to reside in less numerous, more massive and more biased halos. Therefore reproducing the high emissivity of luminous quasars requires BHs accreting at a significant fraction of the Eddington luminosity $(\lambda \gtrsim 0.25)$ to radiate at high radiative efficiencies $(\epsilon \gtrsim 0.15)$ to match the AGN luminosity function and its evolution with redshift. 

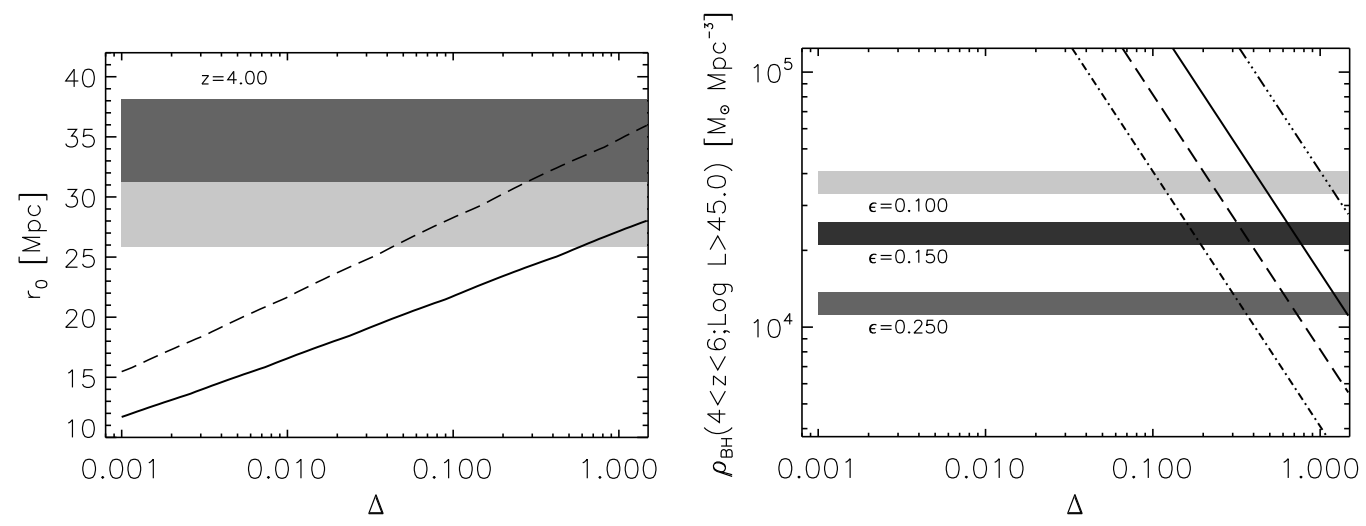

Figure 12: Left panel: predicted $z=4.0$ clustering correlation length $r_{0}$ computed above the minimum survey sensitivity as a function of duty cycle, having adopted the luminosity function derived from the reference model by Shankar et al. (2008b). The solid and long-dashed lines refer to the $r_{0}$ implied by using different bias formulae (see Shankar et al. 2008b). Dark and light shaded bands show the $1 \sigma$ range of the Shen et al. (2007) measurements at these redshifts. Maximal values of the duty cycle predict a clustering strength only marginally consistent with the data at $z=4$. Right panel: horizontal stripes show the integrated black hole mass density above $L=10^{45} \mathrm{erg} \mathrm{s}^{-1}$ and $4.0<z<6$ derived from the AGN luminosity function for three different values of the radiative efficiency, as labeled; triple dot-dashed, solid, long-dashed, and dot-dashed lines indicate the black hole mass density implied by the AGN luminosity function at $z=4.0$ integrated over mass assuming a mean Eddington ratio $\lambda=0.1,0.25,0.5,1$, respectively, as a function of duty cycle $\Delta$. High duty cycles require high radiative efficiencies or low Eddington ratios to reconcile the cumulative AGN emissivity with the black hole mass density in rare halos (see text). Figures taken from Shankar et al. (2008b).

Wyithe and Loeb (2009) also suggested that the strong $z=4$ clustering could be explained by tying quasar activity to recently merged halos, which might have stronger bias (see also Wechsler et al. 2006; Furlanetto and Kamionkowski 2006; Wetzel et al. 2009). However, halos with substantial "excess" bias might be too rare to satisfy duty cycle constraints, and/or the amount of extra bias might be too modest to make any significant difference in the models described by Shankar et al. (2008b). Only numerical simulations will be able to provide significant answers in this direction. 


\section{AN OVERVIEW ON COEVOLUTION MODELS OF BLACK HOLES AND GALAXIES}

\subsection{GALAXY DICHOTOMY AND AGN FEEDBACK}

Galaxies can be classified into two families, which reflect distinct morphological, chemical and evolutionary features. Following, e.g. Kauffmann et al. (2003), Heckman et al. (2004), and Dekel and Birnboim (2006), here are summarized the main pieces of observational evidence supporting this bimodal classification. There is a gap at a color of $u-r \sim 2$, where galaxies naturally divide into a blue and a red sequence. The former are on average younger, less massive and disky (late-type galaxies), while the latter are more massive older and boxy, spheroidal (early-type galaxies). Blue, latetype galaxies dominate the mass function below the knee, $L^{\star}$, of the Schechter function while red, early-type galaxies take over above $L^{\star}$.

There is a remarkable correlation between the colors and luminosities of elliptical galaxies, with brighter galaxies being redder. Values of the elemental ratios $[\alpha / \mathrm{Fe}]$, in particular for $\mathrm{Mg}$, have been found to increase with galactic mass (e.g., Terlevich and Forbes, 2002; Bernardi et al., 2003), implying that the present rate of Supernovae (SN) I is ten times higher than the rate of SNII. The latter is close to being a tracer of the instantaneous star formation rate. These observations thereby constitute significant evidence that massive galaxies formed most of their stars at $z \geq 2-3$ in a relatively short burst. Direct evidence that massive galaxies with $M_{\text {star }} \geq 10^{11} M_{\odot}$ were in place at $z \geq 2$, is provided by recent $K$-band and submillimeter surveys (e.g., Cimatti et al., 2002; Greve et al., 2005). The fundamental plane relation (e.g., Djorgovski and Davis, 1987; Treu, 2004; Shankar and Bernardi, 2009, and references therein) observed up to $z \sim 1$, is also consistent with massive spheroids being old and quiescent.

According to the standard cosmological paradigm of structure formation and evolution, dark matter halos have grown hierarchically, through the merging together of smaller units into ever larger systems. In this scenario, galaxies form inside this hierarchically growing system of halos. However, hierarchical models do not predict the correct abundances of galaxies at high and low redshifts (e.g., Fontana et al., 2006; Marchesini and van Dokkum, 2007) and, at variance with observations, they also tend to generate negative gradients for the $[\alpha / F e]$ abundance with respect to stellar mass, as more $\mathrm{Fe}$ is formed in more massive systems which complete their formation at later times in pure hierarchical models (e.g., Thomas, 1999). 
Therefore in recent years theoretical models of galaxy formation have inserted AGN feedback as a fundamental ingredient for successfully matching several pieces of independent data, from the stellar mass function to chemical abundances (e.g., Wvithe and Loeb, 2003; Granato et al., 2004; Scannapieco and Oh, 2004; Di Matteo et al., 2005; Sazonov et al., 2005; Vittorini et al., 2005; Bower et al., 2006; Cattaneo et al., 2006; Croton et al.,, 2006; De Lucia et al.,, 2006; Hopkins et al., 2006a; Lapi et al., 2006; Shankar et al., 2006; Malbon et al., 2007; Menci et al., 2008; Benson et al., 2007; Monaco et al., 2007; Cook et al., 2009). However, clear and statistically significant evidence for AGN feedback in galaxies is still lacking, despite some significant studies (e.g., Chartas et al., 2003; King and Pounds, 2003; Schawinski et al., 2007; Dai et al., 2008; Shankar et al., 2008c,a).

AGN feedback consists of injection of energy and momentum acting on the gas and dust of the host galaxy. Its implementation in theoretical models differs significantly between different research groups; the energy can be in fact injected into the system in a radiative, thermal, or kinetic mode, and/or at different times during the evolution of the system, and/or under different conditions. These models are, nevertheless, similar in the aims of removing gas from the galaxy, quenching star formation, and turning the galaxy from blue to "red and dead". AGN feedback has also been proven to predict the correct slopes and normalizations of the observed scaling relations between BHs mass and galaxy properties (e.g., Silk and Rees, 1998), such as the stellar dispersion velocity $\sigma$ or $K$-band luminosity, although feedback is not a necessary condition to substantiate such scaling relations (e.g., Miralda-Escudé and Kollmeier, 2005).

The physics of accretion onto the central BH remains, however, poorly understood. The problem of how to remove angular momentum from gas in the inner regions of galaxies allowing it to accrete onto the central $\mathrm{BH}$, is still far from settled. Furthermore, Thompson et al. (2005) pointed out that even if gas is efficiently transferred to the central regions through some global torque, then the high densities will lead all gas to be easily converted into stars at the 1-10 pc scale, leaving no fuel for the $\mathrm{BH}$.

Most SAMs now include some basic recipes to account for $\mathrm{BH}$ growth and the co-evolution with their host galaxies, although the assumptions and results sometimes differ significantly among different groups. Some models predict, for example, a merger dominated growth of BHs at late times, while others state that gas accretion always dominates the evolution. Malbon et al. (2007), within the context of their hierarchical model of galaxy evolution, 
find that while lower mass BHs mainly grow through gas accretion during starbursts, more massive BHs grow first by accretion at higher redshifts, and then predominantly by merging of pre-existing BHs at lower redshifts. This conclusion is not shared by other SAMs, which instead grow all BHs mainly through gas accretion (e.g., Lapi et al. 2006).

SAMs have adopted several different prescriptions for triggering accretion onto the central BHs, such as radiation-drag (e.g., Kawakatu et al., 2003), bar-instabilities (e.g., Begelman et al., 1984), cloud-cloud collisions (e.g., Hopkins and Hernquist, 2006), merging or galaxy interactions (e.g., Cavaliere and Vittorini,, 2000; Di Matteo et al.,, 2005; Croton et al.,, 2006; Hopkins and Hernquist, 2006; Hopkins et al., 2006a), surface density instabilities (e.g., Cen, 2007), stellar feeding (e.g., Miralda-Escudé and Kollmeier, 2005; Ciotti and Ostriker, 2007). Nevertheless, most SAMs provide reasonable matches to the same observables, such as the optical quasar luminosity function, although sometimes their input $\mathrm{BH}$ physics might differ significantly.

While independent, fundamental tests for $\mathrm{BH}$ evolution will probably need to wait for future gravitational wave experiments, such as LISA (the "Laser Interferometer Space Antenna", a joint ESA/NASA mission), important constraints can be set on $\mathrm{BH}$ evolution in ways independent of specific models, via basic accretion and merging models which also include clustering, as already extensively discussed in the previous sections. In this section we instead briefly review some of the main results achieved by several SAMs which attempted to reproduce the overall $\mathrm{BH}$ evolution.

\subsection{UNDERSTANDING THE EVOLUTION AND SHAPE OF THE AGN LUMINOSITY FUNCTION}

The two power-law observed AGN bolometric LF (e.g., ?Shankar et al., $2009 \mathrm{~b}$, and references therein) is an essential tool to probe $\mathrm{BH}$ evolution. In the overall, a significant decline in the AGN luminous density is observed, with the faint-end rapidly steepening at redshifts $z \lesssim 1.2$.

Such an evolution could be described by two extreme scenarios. On the one hand, less massive BHs form continuously over time, to track the population decline to lower luminosities while retaining nearly constant (or slightly declining) Eddington ratios $\lambda \equiv L / M_{\bullet}$, as in Kollmeier et al. (2006). On the other hand, the strong AGN luminosity density redshift evolution could also be explained by a distribution of already massive BHs shining with ever lower luminosities over time, inevitably causing a significant decrease in the overall Eddington ratio distribution. From the simultaneous match to the quasar 
clustering and luminosity function, Wyithe and Loeb (2005) concluded that bright quasars at $z \lesssim 2$ have a lower abundance than expected from the number of merging halos, and discussed how a viable solution would be for quasars to shine at sub-Eddington luminosities. Similar questions arise regarding the steepening of the faint-end of the AGN LF: is it produced by lower-mass BHs shining at the Eddington limit or is it mostly due to highmass $\mathrm{BHs}$ radiating at sub-Eddington luminosities?

Within the framework of their numerically-predicted $\mathrm{BH}$ light curves, Hopkins et al. (2006b) found that the bright-end slope and break luminosity time behavior in the AGN luminosity function, directly reflects the formation rates of BHs shining close to their peak emission following a major merger, while the main contribution to the faint-end is from BHs shining at luminosities far below the peak. This scenario would imply a continuous formation of massive BHs down to very low redshifts driven by major mergers. On other grounds, however, extending major mergers as main triggers for the overall quasar population at all times, might give rise to several problems (e.g., Bournaud et al., 2007; Naab and Ostriker, 2009).

\subsection{THE HIGH-REDSHIFT EVOLUTION: FAST GROWTH OF MAS- SIVE GALAXIES AND BLACK HOLES}

At high- $z$, the main SAMs fail to grow BHs fast and massive enough to reproduce the relatively high number of luminous quasars observed at high- $z$ and their extreme clustering Shen et al. (2007). High-resolution numerical simulations have shown that DM halos grow in a two-phase mode (e.g., Zhao et al., 2003). The inner core and central potential of the host halo is built during a fast, chaotic, merging phase of multiple subhalos after which a longer, smoother accretion phase occurs. During the latter phase, mass is preferentially added to the outskirts of the halo without affecting the inner regions much. This behavior may help in preserving the scaling relations between the central $\mathrm{BH}$ and host galaxy, and, in general, limit the role of environment in the formation of luminous galaxies (e.g., Bournaud et al., 2007; Tinker et al., 2008; Cook et al., 2009). In the DM potential wells, SAMs attempt to speed up the formation of massive galaxies and their BHs by allowing baryons to cool in high clumping factors (e.g., Granato et al., 2004; Maller and Bullock, 2004; Scannapieco et al., 2005 ) and/or cold streams flowing directly to the center (e.g., Dekel and Birnboim, 2006). For example, by using enhanced clumping factors and short formation timescales, Granato et al. (2004), Cirasuolo et al. (2005), Lapi et al. 
(2006) and Shankar et al. (2006), were able to reproduce several dynamical, photometric, and statistical properties of BHs, spheroidal galaxies and AGNs.

At the same time, SAMs need to cope with the difficulty of reproducing the non-linear metallicity behaviors observed in early-type galaxies, with the more massive galaxies being $[\alpha / F e]$-enhanced with respect to the less massive ones. The $F e$ production is roughly proportional to the (delayed) Supernova Ia rate. Thus, reducing the burst timescale in the more massive galaxies, proportionally reduces their amount of $F e$ inducing an $[\alpha / F e]$-enhancement (e.g., Matteucci 1994). In practice, reproducing, and physically explaining, this differential trend in metallicity is a challenge for SAMs. Granato et al. (2004), similarly to other models such as those of Croton et al. (2006), De Lucia et al. (2006), Pipino et al. (2009), naturally find the more massive galaxies to be, on average, the oldest and most metal rich.

However, to get the right $[\alpha / F e]$-enhancement some extreme assumptions need to be made. Granato et al. (2004), for example, allowed for SuperEddington accretion at high redshifts (this assumption has also been used in the recent numerical simulations by Di Matteo et al. 2008), thus boosting $\mathrm{BH}$ growth which more rapidly meet the condition for self-regulation and quench star formation earlier than less massive galaxies. Granato et al. (2006) successfully adopted the same prescriptions to reproduce the bulk of the $850 \mu$ counts (see also Fontanot et al. 2009) observed with the submillimeter array $S C U B A$, a difficult task for hierarchical galaxy formation models (e.g., Granato et al., 2000). By allowing for substantial BH growth during the dust-obscured phase triggered by radiation drag (Kawakatu et al. 2003), they also predicted the correct number counts of AGN sources emitting above a certain X-ray flux limit, as measured by Alexander et al. (2005). Lapi et al. (2006) using the same model to grow BHs to at $z \gtrsim 2$, and a significantly large scatter in the quasar luminosity-halo relation, were then able to reproduce the bright end of the observed AGN luminosity function (see the previous Authors for details). However, a large scatter in the luminosity-halo relation of high- $z$ quasars might be difficult to reconcile with the extreme clustering signal measured for those quasars (see § 77), and more secure data and more detailed modeling is required to set final conclusions.

Similar conclusions on the growth of the most massive BHs at high- $z$ were reached by Marulli et al. (2008; similar results were found by Fontanot et al. 2006 using the MORGANA model). Although their semi-analytical framework, developed on the outputs of the Millennium Simulation, better 
tracks, in principle, DM merger histories than the analytical treatments by Lapi et al. (2006), their final conclusions on the baryonic physics is similar. In fact, while they can reproduce the observed AGN luminosity function at low and intermediate redshifts, at $z>1$ they under-predict the number density of bright $\mathrm{AGN}$, regardless of the $\mathrm{BH}$ mass accretion rate and light curve model assumed for each quasar episode. Despite the considerable freedom in choosing the Eddington ratio distributions, they failed to find a model able to simultaneously match the observed $\mathrm{BH}$ scaling relations, the $\mathrm{BH}$ mass function, and the AGN luminosity function, especially at high redshifts. Also allowing for larger seed BH masses, they were still not able to fit the high- $z$ luminosity function. They interpreted this failure as an indication that the present theoretical framework is itself inadequate to account for the full AGN phenomenon. Analogously to Lapi et al. and Granato et al., Marulli et al. (2008) also suggested that a significant improvement in the model might arise by assuming an accretion efficiency that increases with redshift, for example by forming more concentrated disks in the past, making more efficient the BH feeding (see, e.g., Mo et al. 1998).

\subsection{THE INTERMEDIATE REDSHIFT EVOLUTION: INTERACTIONS, BLACK HOLES RE-ACTIVATIONS, ACTIVITY IN DISKS}

Although efficient, the fast accretion phase occurring during the epoch of rapid merger events in the high-redshift Universe cannot be the only viable formation mode to fully grow all local BHs. For $z \lesssim 1.5$ major mergers become much rarer at galactic scales; fewer new massive BHs are then expected to be formed. The prevailing dynamical events that could still trigger star formation in a galaxy and possible gas accretion onto the central $\mathrm{BH}$, are best described as interactions between galaxies, occurring in the small, dense groups that begin to form at these epochs. The accretion is also expected to be supply-limited, due to progressive dilution of the host gas reservoir and lack of fresh gas resupply from gas-rich mergers.

As discussed in (e.g., Cavaliere and Vittorini, 2000; Hopkins et al., 2008, and references therein), small galactic groups with virial mass $\gtrsim 10^{13} \mathrm{M}_{\odot}$ could provide particularly suitable sites for the hosts to interact with their companions. An interaction of the host with a group companion will perturb the galactic potential, and destabilize a fraction $f$ of the cold gas mass $m(z)$ in the host from kpc scales to the inner regions, possibly triggering a circumnuclear starburst (see, e.g., the numerical simulations by Mihos 1999, and the IR observations by Franceschini et al. 1993) and reactivating the central 
BH to a level of luminosity $L \propto f m(z)$. Direct evidences of star-formation activity at intermediate redshifts connected with clearly interacting galaxies have now been provided by several groups (e.g., Urrutia et al., 2008, and references therein), who also find that a large fraction of AGNs have close companions or show signs of ongoing or recent interactions, although locally and/or at lower luminosities this association between AGN activity and close companions might break down, as discussed in $\S 7$.

Vittorini et al. (2005) have carried out a detailed semi-analytical computation of interactions and minor/major merging of galaxies within the framework of the hierarchical growth of dark matter halos. They found that the strong luminosity decline with redshift of the AGN luminosity function, given by $L \propto f \times m(z)$, is fully accounted for by the continuous exhaustion of gas $m(z)$ driven by galaxy interactions. The highly stochastic nature of the accretion events described by interactions, parameterized in the factor $f$, predicts a largely increasing scatter in the Eddington ratio distributions at lower redshifts, peaked at lower and lower accretion rates, in line with the several recent empirical findings discussed in $\S 5$.

Other possible ways to reproduce the bright end of the quasar luminosity function have been extensively discussed in, e.g., Scannapieco and Oh (2004), and Croton et al. (2006). These models mimic the fall-off and steepening of the quasar luminosity function at low redshifts by assuming that late AGN feedback in massive halos prevents cooling flows in massive halos (the so-called "radio-mode" feedback). More recently, Cavaliere and Menci (2007) have presented the full predictions of their semi-analytic model for the co-evolution of BHs and galaxies, which includes galaxy interactions, among other dynamical triggers. Their model predicts a "bimodal" Eddington ratio distribution, with a peak around $\lambda \sim 0.01$, mainly contributed by low-luminosity AGNs in red galaxies, and a peak at $\lambda \lesssim 1$ contributed by lower mass BHs in bluer, star-forming galaxies.

It is clear, especially after the significant results obtained from SDSS data (e.g., Kauffmann et al., 2003; Kauffmann and Heckman, 2008; Li et al., 2006, 2008), that a significant part of the AGN activity in the local Universe must be triggered by other mechanisms other than major mergers, such as bar instabilities, radiation drag, stellar winds. Moreover, observations have unveiled the presence of AGNs also in isolated disk-dominated galaxies (e.g., Satyapal et al., 2008), as discussed in $\S$ 3.1. AGN feedback might have been possibly efficient due to the lower mass in the central BHs (e.g., Kawakatu and Umemura, 2004; Shankar et al., 2006), and the metal- 
rich gas outflow may be expected to recollapse towards the center on a relatively short timescale, due to the shallower potential wells that characterize the halos hosting disk galaxies today. Tsujimoto (2007) finds that if one includes a double infall of primordial and enriched gas in chemical evolution models of the Galaxy, with the enriched outflow occurring after 1 Gyr since the onset of the star formation, it is possible to explain the observed $\alpha$-element abundances and their non-linear behavior in the Galaxy as a function of iron abundance. This model can also simultaneously reproduce the sharply peaked metal abundance of disk stars against iron abundance.

To sum up, most models agree in associating the luminous, high- $z$ quasars with fast, gas-rich merging events, which rapidly built up the host potential wells and the scaling relations discussed in $\S$ 2. A longer and milder activity phase has probably occurred at lower redshifts, during which in-situ and milder dynamical events have regulated the late evolution in the AGN/BH population. However, as discussed in this section, many details of this basic picture are still missing, and are currently the subject of intense investigation.

\section{Conclusions}

This Review addresses the main topics and aims concerning the evolution of the $\mathrm{BH}$ population, and discusses several ways to constrain it in ways independent of specific models of galaxy evolution. The main results can be listed as follows.

- The local BHMF can be now rather accurately assessed. In more detail, the BHMF hosted in early-type galaxies can be obtained on exploiting the velocity dispersion or luminosity functions of host galaxies, coupled with the $M_{\mathrm{BH}}-\sigma$ or $M_{\mathrm{BH}}-L_{\mathrm{sph}}$ relationships, respectively, yielding similar results within $\sim 30 \%$ accuracy. The contribution from $\mathrm{BH}$ hosted by late-type galaxies is more uncertain, and is mostly confined to the low mass end of the BHMF. The overall $\mathrm{BH}$ mass density amounts to $\rho_{\mathrm{BH}}=(3-5) \times 10^{5} M_{\odot} / \mathrm{Mpc}^{3}(\S[3)$.

- The match both between the local BH mass density and the one predicted by integrating the bolometri AGN luminosity function (Soltan 1982) yields interesting constraints for a mass to radiation conversion

efficiency $\epsilon \lesssim 0.1$, although systematic uncertainties on bolometric corrections, luminosity functions, and the local BHMF prevent more precise estimates $(\S$ 4). 
- The average accretion history of BHs tracks the average SFR, with a proportionality constant of the order of the local one. Several works have empirically proven that the ratio between the degree of evolution in the local relations is relatively mild, suggesting that the bulk of the $\mathrm{BH}$ and host galaxy populations should have mostly co-evolved, at least up to $z \lesssim 2(\S \square)$.

- The shape of the local BHMF yields additional interesting constraints on the typical Eddington ratio $\lambda \sim 0.5$. The alternative possibility that most of the mass locked in BHs in the local Universe has been accumulated by "dark" accretion (i.e., accretion undetectable by either optical or hard X-ray surveys, as in the case of BH coalescence), is severely constrained by the above results. In order to make room for this possibility one has to assume that the radiative efficiency during the visible $\mathrm{AGN}$ phases is at the theoretical maximum of $\epsilon \simeq 0.3$ 0.4. But even in this case (unless the bolometric correction is far lower than currently estimated) the contribution of radiative accretion to the local $\mathrm{BH}$ mass density is $\geq 25 \%$, and one is left with the problem of fine tuning the radiative and non-radiative contributions in order not to break down the match with the local BHMF obtained with radiative accretion alone. One would also face the problem of accounting for the tight relationships between $\mathrm{BH}$ mass and mass or velocity dispersion of the spheroidal host, naturally explained by feedback associated to radiative accretion. The analysis of the accretion history highlights that the most massive BHs (associated to bright optical quasars) accreted their mass faster and at higher redshifts (typically at $z>1.5$ ), while the lower mass BHs have mostly grown at $z<1.5$ ( $\S \S 5$ and 6 ).

- Quasar clustering provides additional, independent constraints on the main parameters governing the $\mathrm{BH}$ evolution. It has been shown how AGN clustering can set important constraint on the possible redshift and/or mass dependence of the typical $\lambda$ and $\epsilon$, thus helping us in discriminating among a number of successful models. Clustering is in fact a direct measure of the masses, and therefore number densities, of the halos hosting the quasars. Matching the quasar host number density with the one derived from accretion models (Eq. [11]), in turn yields interesting constraints on radiative efficiency and Eddington ratios as a function of time and/or BH mass ( $\S$ [7). 
- Most galaxy formation models now include some basic recipes to account for BH growth and the coevolution with their host galaxies, although it has been discussed in this Review that the assumptions and results sometimes differ significantly among different groups ( $(8)$. The model-independent results discussed above will help shedding light on the true physical mechanisms which regulate $\mathrm{BH}$ growth across time.

\section{Acknowledgments}

I would like to warmly thank here many collaborators whom I had the pleasure to work with over the years, Mariangela Bernardi, Silvia Bonoli, Alfonso Cavaliere, Martin Crocce, Xinyu Dai, Luigi Danese, Gianfranco De Zotti, Laura Ferrarese, Gianluigi Granato, Zoltan Haiman, Andrea Lapi, Cheng Li, Federico Marulli, Smita Mathur, Jordi Miralda-Escudé, Jorge Moreno, Paolo Salucci, Yue Shen, Ravi K. Sheth, Gregory R. Sivakoff, Valerio Vittorini, David H. Weinberg. I acknowledge the Alexander von Humboldt Foundation and NASA Grant NNG05GH77G for support. I finally thank Lucas Bakker for assistance during the editing of the manuscript.

\section{References}

Alexander, D. M., Bauer, F. E., Chapman, S. C., Smail, I., Blain, A. W., Brandt, W. N., Ivison, R. J., Oct. 2005. The X-Ray Spectral Properties of SCUBA Galaxies. ApJ 632, 736-750.

Aller, M. C., Richstone, D., Dec. 2002. The Cosmic Density of Massive Black Holes from Galaxy Velocity Dispersions. AJ 124, 3035-3041.

Aller, M. C., Richstone, D. O., Aug. 2007. Host Galaxy Bulge Predictors of Supermassive Black Hole Mass. ApJ 665, 120-156.

Bardeen, J. M., Press, W. H., Teukolsky, S. A., Dec. 1972. Rotating Black Holes: Locally Nonrotating Frames, Energy Extraction, and Scalar Synchrotron Radiation. ApJ 178, 347-370.

Barth, A. J., Nov. 2004. Black hole masses in active galaxies 222, 3-8.

Barway, S., Kembhavi, A., Jun. 2007. A Supermassive Black Hole Fundamental Plane for Ellipticals. ApJ 662, L67-L70. 
Batcheldor, D., Marconi, A., Merritt, D., Axon, D. J., Jul. 2007. How Special Are Brightest Cluster Galaxies? The Impact of Near-Infrared Luminosities on Scaling Relations for BCGs. ApJ 663, L85-L88.

Begelman, M. C., Blandford, R. D., Rees, M. J., Apr. 1984. Theory of extragalactic radio sources. Reviews of Modern Physics 56, 255-351.

Benson, A. J., Džanović, D., Frenk, C. S., Sharples, R., Aug. 2007. Luminosity and stellar mass functions of discs and spheroids in the SDSS and the supermassive black hole mass function. MNRAS 379, 841-866.

Bentz, M. C., Peterson, B. M., Pogge, R. W., Vestergaard, M., Onken, C. A., Jun. 2006. The Radius-Luminosity Relationship for Active Galactic Nuclei: The Effect of Host-Galaxy Starlight on Luminosity Measurements. ApJ 644, 133-142.

Bernardi, M., Sheth, R. K., Annis, J., Burles, S., Eisenstein, D. J., Finkbeiner, D. P., Hogg, D. W., Lupton, R. H., Schlegel, D. J., SubbaRao, M., Bahcall, N. A., Blakeslee, J. P., Brinkmann, J., Castander, F. J., Connolly, A. J., Csabai, I., Doi, M., Fukugita, M., Frieman, J., Heckman, T., Hennessy, G. S., Ivezić, Ž., Knapp, G. R., Lamb, D. Q., McKay, T., Munn, J. A., Nichol, R., Okamura, S., Schneider, D. P., Thakar, A. R., York, D. G., Apr. 2003. Early-Type Galaxies in the Sloan Digital Sky Survey. I. The Sample. AJ 125, 1817-1848.

Berti, E., Volonteri, M., Sep. 2008. Cosmological Black Hole Spin Evolution by Mergers and Accretion. ApJ 684, 822-828.

Boeker, T., Aug. 2007. Nuclear Star Clusters across the Hubble Sequence. ArXiv e-prints.

Bonoli, S., Marulli, F., Springel, V., White, S. D. M., Branchini, E., Moscardini, L., Nov. 2008. Modeling the cosmological co-evolution of supermassive black holes and galaxies: II. The clustering of quasars and their dark environment. ArXiv:0812.0003.

Bournaud, F., Jog, C. J., Combes, F., Dec. 2007. Multiple minor mergers: formation of elliptical galaxies and constraints for the growth of spiral disks. A\&A 476, 1179-1190. 
Bower, R. G., Benson, A. J., Malbon, R., Helly, J. C., Frenk, C. S., Baugh, C. M., Cole, S., Lacey, C. G., Aug. 2006. Breaking the hierarchy of galaxy formation. MNRAS 370, 645-655.

Boyle, B. J., Shanks, T., Croom, S. M., Smith, R. J., Miller, L., Loaring, N., Heymans, C., Oct. 2000. The 2dF QSO Redshift Survey - I. The optical luminosity function of quasi-stellar objects. MNRAS 317, 1014-1022.

Bundy, K., Fukugita, M., Ellis, R. S., Targett, T. A., Belli, S., Kodama, T., Jun. 2009. The Greater Impact of Mergers on the Growth of Massive Galaxies: Implications for Mass Assembly and Evolution since z sime 1. ApJ 697, 1369-1383.

Cao, X., Li, F., Oct. 2008. Rapidly spinning massive black holes in active galactic nuclei: evidence from the black hole mass function. MNRAS 390, 561-566.

Cattaneo, A., Best, P. N., May 2009. On the jet contribution to the active galactic nuclei cosmic energy budget. MNRAS 395, 518-523.

Cattaneo, A., Dekel, A., Devriendt, J., Guiderdoni, B., Blaizot, J., Aug. 2006. Modelling the galaxy bimodality: shutdown above a critical halo mass. MNRAS 370, 1651-1665.

Cavaliere, A., Menci, N., Jul. 2007. Bimodal Active Nuclei in Bimodal Galaxies. ApJ 664, 47-52.

Cavaliere, A., Morrison, P., Wood, K., Dec. 1971. On Quasar Evolution. ApJ $170,223-+$.

Cavaliere, A., Vittorini, V., Nov. 2000. The Fall of the Quasar Population. ApJ 543, 599-610.

Cen, R., Jan. 2007. A Physical Framework for Grand Unification of Galaxies and Active Galactic Nuclei. I. Origin of the Black Hole Mass-Bulge Velocity Dispersion Relation. ApJ 654, L37-L40.

Chartas, G., Brandt, W. N., Gallagher, S. C., Sep. 2003. XMM-Newton Reveals the Quasar Outflow in PG 1115+080. ApJ 595, 85-93. 
Cimatti, A., Pozzetti, L., Mignoli, M., Daddi, E., Menci, N., Poli, F., Fontana, A., Renzini, A., Zamorani, G., Broadhurst, T., Cristiani, S., D’Odorico, S., Giallongo, E., Gilmozzi, R., Aug. 2002. The K20 survey. IV. The redshift distribution of $K_{s}<20$ galaxies: A test of galaxy formation models. A\&A 391, L1-L5.

Ciotti, L., Ostriker, J. P., Aug. 2007. Radiative Feedback from Massive Black Holes in Elliptical Galaxies: AGN Flaring and Central Starburst Fueled by Recycled Gas. ApJ 665, 1038-1056.

Cirasuolo, M., Shankar, F., Granato, G. L., De Zotti, G., Danese, L., Aug. 2005. Dynamical and Photometric Imprints of Feedback Processes on the Formation and Evolution of E/S0 Galaxies. ApJ 629, 816-824.

Coil, A. L., Georgakakis, A., Newman, J. A., Cooper, M. C., Croton, D., Davis, M., Koo, D. C., Laird, E. S., Nandra, K., Weiner, B. J., Willmer, C. N. A., Yan, R., Feb. 2009. AEGIS: The Clustering of X-ray AGN Relative to Galaxies at $\mathrm{z}^{\sim} 1$. ArXiv e-prints.

Coil, A. L., Hennawi, J. F., Newman, J. A., Cooper, M. C., Davis, M., Jan. 2007. The DEEP2 Galaxy Redshift Survey: Clustering of Quasars and Galaxies at $\mathrm{z}=1$. ApJ 654, 115-124.

Constantin, A., Vogeley, M. S., Oct. 2006. The Clustering of Low-Luminosity Active Galactic Nuclei. ApJ 650, 727-748.

Cook, M., Lapi, A., Granato, G. L., Mar. 2009. Two-Phase Galaxy Formation. ArXiv:0903.2390.

Coppin, K. E. K., Swinbank, A. M., Neri, R., Cox, P., Alexander, D. M., Smail, I., Page, M. J., Stevens, J. A., Knudsen, K. K., Ivison, R. J., Beelen, A., Bertoldi, F., Omont, A., Sep. 2008. Testing the evolutionary link between submillimetre galaxies and quasars: CO observations of QSOs at $\mathrm{z} \sim 2$. MNRAS 389, 45-62.

Côté, P., Piatek, S., Ferrarese, L., Jordán, A., Merritt, D., Peng, E. W., Haşegan, M., Blakeslee, J. P., Mei, S., West, M. J., Milosavljević, M., Tonry, J. L., Jul. 2006. The ACS Virgo Cluster Survey. VIII. The Nuclei of Early-Type Galaxies. ApJS 165, 57-94. 
Croom, S. M., Boyle, B. J., Shanks, T., Smith, R. J., Miller, L., Outram, P. J., Loaring, N. S., Hoyle, F., da Ângela, J., Jan. 2005. The 2dF QSO Redshift Survey - XIV. Structure and evolution from the two-point correlation function. MNRAS 356, 415-438.

Croton, D. J., Springel, V., White, S. D. M., De Lucia, G., Frenk, C. S., Gao, L., Jenkins, A., Kauffmann, G., Navarro, J. F., Yoshida, N., Jan. 2006. The many lives of active galactic nuclei: cooling flows, black holes and the luminosities and colours of galaxies. MNRAS 365, 11-28.

da Ângela, J., Shanks, T., Croom, S. M., Weilbacher, P., Brunner, R. J., Couch, W. J., Miller, L., Myers, A. D., Nichol, R. C., Pimbblet, K. A., de Propris, R., Richards, G. T., Ross, N. P., Schneider, D. P., Wake, D., Jan. 2008. The 2dF-SDSS LRG and QSO survey: QSO clustering and the L-z degeneracy. MNRAS 383, 565-580.

Dai, X., Shankar, F., Sivakoff, G. R., Jan. 2008. 2MASS Reveals a Large Intrinsic Fraction of BALQSOs. ApJ 672, 108-114.

Dasyra, K. M., Tacconi, L. J., Davies, R. I., Genzel, R., Lutz, D., Peterson, B. M., Veilleux, S., Baker, A. J., Schweitzer, M., Sturm, E., Mar. 2007. Host Dynamics and Origin of Palomar-Green QSOs. ApJ 657, 102-115.

De Lucia, G., Springel, V., White, S. D. M., Croton, D., Kauffmann, G., Feb. 2006. The formation history of elliptical galaxies. MNRAS 366, 499-509.

Dekel, A., Birnboim, Y., May 2006. Galaxy bimodality due to cold flows and shock heating. MNRAS 368, 2-20.

Di Matteo, T., Colberg, J., Springel, V., Hernquist, L., Sijacki, D., Mar. 2008. Direct Cosmological Simulations of the Growth of Black Holes and Galaxies. ApJ 676, 33-53.

Di Matteo, T., Springel, V., Hernquist, L., Feb. 2005. Energy input from quasars regulates the growth and activity of black holes and their host galaxies. Nature 433, 604-607.

Djorgovski, S., Davis, M., Feb. 1987. Fundamental properties of elliptical galaxies. ApJ 313, 59-68. 
Driver, S. P., Popescu, C. C., Tuffs, R. J., Liske, J., Graham, A. W., Allen, P. D., de Propris, R., Aug. 2007. The Millennium Galaxy Catalogue: the B-band attenuation of bulge and disc light and the implied cosmic dust and stellar mass densities. MNRAS 379, 1022-1036.

Elitzur, M., Aug. 2008. The toroidal obscuration of active galactic nuclei. New Astronomy Review 52, 274-288.

Elvis, M., Wilkes, B. J., McDowell, J. C., Green, R. F., Bechtold, J., Willner, S. P., Oey, M. S., Polomski, E., Cutri, R., Nov. 1994. Atlas of quasar energy distributions. ApJS 95, 1-68.

Ferrarese, L., Oct. 2002a. Beyond the Bulge: A Fundamental Relation between Supermassive Black Holes and Dark Matter Halos. ApJ 578, 90-97.

Ferrarese, L., 2002b. Black Hole Demographics. In: Lee, C.-H., Chang, H.-Y. (Eds.), Current high-energy emission around black holes. pp. 3-8.

Ferrarese, L., Côté, P., Jordán, A., Peng, E. W., Blakeslee, J. P., Piatek, S., Mei, S., Merritt, D., Milosavljević, M., Tonry, J. L., West, M. J., Jun. 2006. The ACS Virgo Cluster Survey. VI. Isophotal Analysis and the Structure of Early-Type Galaxies. ApJS 164, 334-434.

Ferrarese, L., Ford, H., Feb. 2005. Supermassive Black Holes in Galactic Nuclei: Past, Present and Future Research. Space Science Reviews 116, 523-624.

Ferrarese, L., Merritt, D., Aug. 2000. A Fundamental Relation between Supermassive Black Holes and Their Host Galaxies. ApJ 539, L9-L12.

Fontana, A., Salimbeni, S., Grazian, A., Giallongo, E., Pentericci, L., Nonino, M., Fontanot, F., Menci, N., Monaco, P., Cristiani, S., Vanzella, E., de Santis, C., Gallozzi, S., Dec. 2006. The Galaxy mass function up to z $=4$ in the GOODS-MUSIC sample: into the epoch of formation of massive galaxies. A\&A 459, 745-757.

Fontanot, F., Cristiani, S., Monaco, P., Nonino, M., Vanzella, E., Brandt, W. N., Grazian, A., Mao, J., Jan. 2007. The luminosity function of highredshift quasi-stellar objects. A combined analysis of GOODS and SDSS. A\&A 461, 39-48. 
Fontanot, F., Somerville, R. S., Silva, L., Monaco, P., Skibba, R., Jan. 2009. Evaluating and improving semi-analytic modelling of dust in galaxies based on radiative transfer calculations. MNRAS 392, 553-569.

Franceschini, A., Martin-Mirones, J. M., Danese, L., de Zotti, G., Sep. 1993. Hard and Soft X-Ray Selected Active Galactic Nuclei - Two Distinct Populations. MNRAS 264, 35--+.

Francke, H., Gawiser, E., Lira, P., Treister, E., Virani, S., Cardamone, C., Urry, C. M., van Dokkum, P., Quadri, R., Jan. 2008. Clustering of Intermediate-Luminosity X-Ray-Selected Active Galactic Nuclei at z $\sim 3$. ApJ 673, L13-L16.

Fukugita, M., Hogan, C. J., Peebles, P. J. E., Aug. 1998. The Cosmic Baryon Budget. ApJ 503, 518-+.

Furlanetto, S. R., Kamionkowski, M., Feb. 2006. Pair correlations and merger bias. MNRAS 366, 529-536.

Gadotti, D. A., Oct. 2008. Structural Properties of Pseudo-Bulges, Classical Bulges and Elliptical Galaxies: an SDSS Perspective. ArXiv e-prints.

Gallo, E., Treu, T., Jacob, J., Woo, J.-H., Marshall, P. J., Antonucci, R., Jun. 2008. AMUSE-Virgo. I. Supermassive Black Holes in Low-Mass Spheroids. ApJ 680, 154-168.

Gammie, C. F., Shapiro, S. L., McKinney, J. C., Feb. 2004. Black Hole Spin Evolution. ApJ 602, 312-319.

Gebhardt, K., et al., Aug. 2000. A Relationship between Nuclear Black Hole Mass and Galaxy Velocity Dispersion. ApJ 539, L13-L16.

Gebhardt, K., Lauer, T. R., Kormendy, J., Pinkney, J., Bower, G. A., Green, R., Gull, T., Hutchings, J. B., Kaiser, M. E., Nelson, C. H., Richstone, D., Weistrop, D., Nov. 2001. M33: A Galaxy with No Supermassive Black Hole. AJ 122, 2469-2476.

Genzel, R., Karas, V., Apr. 2007. The Galactic Center 238, 173-180.

Ghez, A. M., Duchêne, G., Matthews, K., Hornstein, S. D., Tanner, A., Larkin, J., Morris, M., Becklin, E. E., Salim, S., Kremenek, T., Thompson, 
D., Soifer, B. T., Neugebauer, G., McLean, I., Apr. 2003. The First Measurement of Spectral Lines in a Short-Period Star Bound to the Galaxy's Central Black Hole: A Paradox of Youth. ApJ 586, L127-L131.

Ghosh, H., Mathur, S., Fiore, F., Ferrarese, L., Nov. 2008. Low-Level Nuclear Activity in Nearby Spiral Galaxies. ApJ 687, 216-229.

Graham, A. W., Aug. 2007. The black hole mass - spheroid luminosity relation. MNRAS 379, 711-722.

Graham, A. W., Jun. 2008. Fundamental Planes and the Barless $M_{b h}-\sigma$ Relation for Supermassive Black Holes. ApJ 680, 143-153.

Graham, A. W., Driver, S. P., Allen, P. D., Liske, J., Jun. 2007. The Millennium Galaxy Catalogue: the local supermassive black hole mass function in early- and late-type galaxies. MNRAS 378, 198-210.

Graham, A. W., Erwin, P., Caon, N., Trujillo, I., Dec. 2001. A Correlation between Galaxy Light Concentration and Supermassive Black Hole Mass. ApJ 563, L11-L14.

Granato, G. L., De Zotti, G., Silva, L., Bressan, A., Danese, L., Jan. 2004. A Physical Model for the Coevolution of QSOs and Their Spheroidal Hosts. ApJ 600, 580-594.

Granato, G. L., Lacey, C. G., Silva, L., Bressan, A., Baugh, C. M., Cole, S., Frenk, C. S., Oct. 2000. The Infrared Side of Galaxy Formation. I. The Local Universe in the Semianalytical Framework. ApJ 542, 710-730.

Granato, G. L., Silva, L., Lapi, A., Shankar, F., De Zotti, G., Danese, L., May 2006. The growth of the nuclear black holes in submillimetre galaxies. MNRAS 368, L72-L76.

Grazian, A., Negrello, M., Moscardini, L., Cristiani, S., Haehnelt, M. G., Matarrese, S., Omizzolo, A., Vanzella, E., Feb. 2004. The AsiagoESO/RASS QSO Survey. III. Clustering Analysis and Theoretical Interpretation. AJ 127, 592-605.

Greve, T. R., Bertoldi, F., Smail, I., Neri, R., Chapman, S. C., Blain, A. W., Ivison, R. J., Genzel, R., Omont, A., Cox, P., Tacconi, L., Kneib, J.P., May 2005. An interferometric CO survey of luminous submillimetre galaxies. MNRAS 359, 1165-1183. 
Gultekin, K., Richstone, D. O., Gebhardt, K., Lauer, T. R., Tremaine, S., Aller, M. C., Bender, R., Dressler, A., Faber, S. M., Filippenko, A. V., Green, R., Ho, L. C., Kormendy, J., Magorrian, J., Pinkney, J., Siopis, C., Mar. 2009. The M-sigma and M-L Relations in Galactic Bulges and Determinations of their Intrinsic Scatter. ArXiv:0903.4897.

Haehnelt, M. G., Natarajan, P., Rees, M. J., Nov. 1998. High-redshift galaxies, their active nuclei and central black holes. MNRAS 300, 817-827.

Haiman, Z., Ciotti, L., Ostriker, J. P., May 2004. Reasoning From Fossils: Learning from the Local Black Hole Population about the Evolution of Quasars. ApJ 606, 763-773.

Haiman, Z., Hui, L., Jan. 2001. Constraining the Lifetime of Quasars from Their Spatial Clustering. ApJ 547, 27-38.

Haiman, Z., Quataert, E., Aug. 2004. The Formation and Evolution of the First Massive Black Holes 308, 147-+.

Häring, N., Rix, H.-W., Apr. 2004. On the Black Hole Mass-Bulge Mass Relation. ApJ 604, L89-L92.

Heckman, T. M., Kauffmann, G., Brinchmann, J., Charlot, S., Tremonti, C., White, S. D. M., Sep. 2004. Present-Day Growth of Black Holes and Bulges: The Sloan Digital Sky Survey Perspective. ApJ 613, 109-118.

Hennawi, J. F., Strauss, M. A., Oguri, M., Inada, N., Richards, G. T., Pindor, B., Schneider, D. P., Becker, R. H., Gregg, M. D., Hall, P. B., Johnston, D. E., Fan, X., Burles, S., Schlegel, D. J., Gunn, J. E., Lupton, R. H., Bahcall, N. A., Brunner, R. J., Brinkmann, J., Jan. 2006. Binary Quasars in the Sloan Digital Sky Survey: Evidence for Excess Clustering on Small Scales. AJ 131, 1-23.

Hickox, R. C., Jones, C., Forman, W. R., Murray, S. S., Kochanek, C. S., Eisenstein, D., Jannuzi, B. T., Dey, A., Brown, M. J. I., Stern, D., Eisenhardt, P. R., Gorjian, V., Brodwin, M., Narayan, R., Cool, R. J., Kenter, A., Caldwell, N., Anderson, M. E., May 2009. Host Galaxies, Clustering, Eddington Ratios, and Evolution of Radio, X-Ray, and Infrared-Selected AGNs. ApJ 696, 891-919. 
Hopkins, P. F., Hernquist, L., Sep. 2006. Fueling Low-Level AGN Activity through Stochastic Accretion of Cold Gas. ApJS 166, 1-36.

Hopkins, P. F., Hernquist, L., Sep. 2008. Quasars Are Not Light-Bulbs: Testing Models of Quasar Lifetimes with the Observed Eddington Ratio Distribution. ArXiv e-prints.

Hopkins, P. F., Hernquist, L., Mar. 2009. A Characteristic Division Between the Fueling of Quasars and Seyferts: Five Simple Tests. ApJ 694, 599-609.

Hopkins, P. F., Hernquist, L., Cox, T. J., Di Matteo, T., Robertson, B., Springel, V., Mar. 2006a. A Unified, Merger-driven Model of the Origin of Starbursts, Quasars, the Cosmic X-Ray Background, Supermassive Black Holes, and Galaxy Spheroids. ApJS 163, 1-49.

Hopkins, P. F., Hernquist, L., Cox, T. J., Kereš, D., Apr. 2008. A Cosmological Framework for the Co-Evolution of Quasars, Supermassive Black Holes, and Elliptical Galaxies. I. Galaxy Mergers and Quasar Activity. ApJS 175, 356-389.

Hopkins, P. F., Hernquist, L., Cox, T. J., Robertson, B., Di Matteo, T., Springel, V., Mar. 2006b. The Evolution in the Faint-End Slope of the Quasar Luminosity Function. ApJ 639, 700-709.

Hopkins, P. F., Hernquist, L., Cox, T. J., Robertson, B., Krause, E., Nov. 2007a. An Observed Fundamental Plane Relation for Supermassive Black Holes. ApJ 669, 67-73.

Hopkins, P. F., Richards, G. T., Hernquist, L., Jan. 2007b. An Observational Determination of the Bolometric Quasar Luminosity Function. ApJ 654, 731-753.

Hopkins, P. F., Robertson, B., Krause, E., Hernquist, L., Cox, T. J., Nov. 2006c. An Upper Limit to the Degree of Evolution between Supermassive Black Holes and Their Host Galaxies. ApJ 652, 107-111.

Hosokawa, T., Sep. 2002. Constraining the Lifetime of Quasars with the Present-Day Mass Function of Supermassive Black Holes. ApJ 576, 75-80.

Hu, J., Jun. 2008. The black hole mass-stellar velocity dispersion correlation: bulges versus pseudo-bulges. MNRAS 386, 2242-2252. 
Hughes, S. A., Blandford, R. D., Mar. 2003. Black Hole Mass and Spin Coevolution by Mergers. ApJ 585, L101-L104.

Islam, R. R., Taylor, J. E., Silk, J., Oct. 2004. Massive black hole remnants of the first stars - I. Abundance in present-day galactic haloes. MNRAS $354,427-442$.

Kaspi, S., Smith, P. S., Netzer, H., Maoz, D., Jannuzi, B. T., Giveon, U., Apr. 2000. Reverberation Measurements for 17 Quasars and the Size-MassLuminosity Relations in Active Galactic Nuclei. ApJ 533, 631-649.

Kauffmann, G., Heckman, T. M., Dec. 2008. Feast and Famine: Regulation of Black Hole Growth in Low Redshift Galaxies. ArXiv e-prints.

Kauffmann, G., Heckman, T. M., Tremonti, C., Brinchmann, J., Charlot, S., White, S. D. M., Ridgway, S. E., Brinkmann, J., Fukugita, M., Hall, P. B., Ivezić, Ž., Richards, G. T., Schneider, D. P., Dec. 2003. The host galaxies of active galactic nuclei. MNRAS 346, 1055-1077.

Kawakatu, N., Umemura, M., Jan. 2004. Why Are Massive Black Holes Small in Disk Galaxies? ApJ 601, L21-L24.

Kawakatu, N., Umemura, M., Mori, M., Jan. 2003. Protoquasars: Physical States and Observable Properties. ApJ 583, 85-91.

King, A. R., Pounds, K. A., Oct. 2003. Black hole winds. MNRAS 345, $657-659$.

King, A. R., Pringle, J. E., Nov. 2006. Growing supermassive black holes by chaotic accretion. MNRAS 373, L90-L92.

Kollmeier, J. A., Onken, C. A., Kochanek, C. S., Gould, A., Weinberg, D. H., Dietrich, M., Cool, R., Dey, A., Eisenstein, D. J., Jannuzi, B. T., Le Floc'h, E., Stern, D., Sep. 2006. Black Hole Masses and Eddington Ratios at $0.3<z<4$. ApJ 648, 128-139.

Kormendy, J., Bender, R., Feb. 2009. Correlations between Supermassive Black Holes, Velocity Dispersions, and Mass Deficits in Elliptical Galaxies with Cores. ApJ 691, L142-L146.

Kormendy, J., Gebhardt, K., Richstone, D., May 2000. The $M--L$ bulge Correlation 32, 702--+. 
Kormendy, J., Richstone, D., 1995. Inward Bound-The Search For Supermassive Black Holes In Galactic Nuclei. ARA\&A 33, 581-+.

Lapi, A., Shankar, F., Mao, J., Granato, G. L., Silva, L., De Zotti, G., Danese, L., Oct. 2006. Quasar Luminosity Functions from Joint Evolution of Black Holes and Host Galaxies. ApJ 650, 42-56.

Lauer, T. R., Faber, S. M., Richstone, D., Gebhardt, K., Tremaine, S., Postman, M., Dressler, A., Aller, M. C., Filippenko, A. V., Green, R., Ho, L. C., Kormendy, J., Magorrian, J., Pinkney, J., Jun. 2007a. The Masses of Nuclear Black Holes in Luminous Elliptical Galaxies and Implications for the Space Density of the Most Massive Black Holes. ApJ 662, 808-834.

Lauer, T. R., Tremaine, S., Richstone, D., Faber, S. M., Nov. 2007b. Selection Bias in Observing the Cosmological Evolution of the $M_{\odot}-\sigma$ and $M_{\odot}-L$ Relationships. ApJ 670, 249-260.

Laurikainen, E., Salo, H., Buta, R., Knapen, J. H., Jan. 2009. Bars, ovals and lenses in early-type disk galaxies: probes of galaxy evolution. ArXiv:0901.0641.

Li, C., Kauffmann, G., Heckman, T. M., White, S. D. M., Jing, Y. P., Apr. 2008. Interactions, star formation and AGN activity. MNRAS 385, 19151922.

Li, C., Kauffmann, G., Wang, L., White, S. D. M., Heckman, T. M., Jing, Y. P., Dec. 2006. The clustering of narrow-line AGN in the local Universe. MNRAS 373, 457-468.

Lidz, A., Hopkins, P. F., Cox, T. J., Hernquist, L., Robertson, B., Apr. 2006. The Luminosity Dependence of Quasar Clustering. ApJ 641, 41-49.

Lynden-Bell, D., Aug. 1969. Galactic Nuclei as Collapsed Old Quasars. Nature $223,690-+$.

Magorrian, J., Tremaine, S., Richstone, D., Bender, R., Bower, G., Dressler, A., Faber, S. M., Gebhardt, K., Green, R., Grillmair, C., Kormendy, J., Lauer, T., Jun. 1998. The Demography of Massive Dark Objects in Galaxy Centers. AJ 115, 2285-2305. 
Malbon, R. K., Baugh, C. M., Frenk, C. S., Lacey, C. G., Dec. 2007. Black hole growth in hierarchical galaxy formation. MNRAS 382, 1394-1414.

Maller, A. H., Bullock, J. S., Dec. 2004. Multiphase galaxy formation: highvelocity clouds and the missing baryon problem. MNRAS 355, 694-712.

Maraschi, L., 2002. From the Spectral Energy Distributions of Blazars to a Unified Scenario. In: Giommi, P., Massaro, E., Palumbo, G. (Eds.), Blazar Astrophysics with BeppoSAX and Other Observatories. pp. 237-+.

Marchesini, D., van Dokkum, P. G., Jul. 2007. Assessing the Predictive Power of Galaxy Formation Models: A Comparison of Predicted and Observed Rest-Frame Optical Luminosity Functions at $2.0 \leq z \leq 3.3$. ApJ 663, L89-L92.

Marconi, A., Hunt, L. K., May 2003. The Relation between Black Hole Mass, Bulge Mass, and Near-Infrared Luminosity. ApJ 589, L21-L24.

Marconi, A., Risaliti, G., Gilli, R., Hunt, L. K., Maiolino, R., Salvati, M., Jun. 2004. Local supermassive black holes, relics of active galactic nuclei and the X-ray background. MNRAS 351, 169-185.

Martini, P., Weinberg, D. H., Jan. 2001. Quasar Clustering and the Lifetime of Quasars. ApJ 547, 12-26.

Marulli, F., Bonoli, S., Branchini, E., Gilli, R., Moscardini, L., Springel, V., Apr. 2009. The spatial distribution of X-ray selected AGN in the Chandra deep fields: a theoretical perspective. ArXiv:0904.0256.

Marulli, F., Bonoli, S., Branchini, E., Moscardini, L., Springel, V., Apr. 2008. Modelling the cosmological co-evolution of supermassive black holes and galaxies - I. BH scaling relations and the AGN luminosity function. MNRAS 385, 1846-1858.

Matteucci, F., Aug. 1994. Abundance ratios in ellipticals and galaxy formation. A\&A 288, 57-64.

McLure, R. J., Dunlop, J. S., Oct. 2001. The black hole masses of Seyfert galaxies and quasars. MNRAS 327, 199-207.

McLure, R. J., Dunlop, J. S., Aug. 2004. The cosmological evolution of quasar black hole masses. MNRAS 352, 1390-1404. 
McLure, R. J., Jarvis, M. J., Targett, T. A., Dunlop, J. S., Best, P. N., May 2006. On the evolution of the black hole: spheroid mass ratio. MNRAS $368,1395-1403$.

Menci, N., Cavaliere, A., Fontana, A., Giallongo, E., Poli, F., Vittorini, V., Mar. 2004. Early Hierarchical Formation of Massive Galaxies Triggered by Interactions. ApJ 604, 12-17.

Menci, N., Fiore, F., Puccetti, S., Cavaliere, A., Oct. 2008. The Blast Wave Model for AGN Feedback: Effects on AGN Obscuration. ApJ 686, 219-229.

Menci, N., Fontana, A., Giallongo, E., Grazian, A., Salimbeni, S., Aug. 2006. The Abundance of Distant and Extremely Red Galaxies: The Role of AGN Feedback in Hierarchical Models. ApJ 647, 753-762.

Merloni, A., Heinz, S., Aug. 2008. A synthesis model for AGN evolution: supermassive black holes growth and feedback modes. MNRAS 388, 10111030.

Merloni, A., Rudnick, G., Di Matteo, T., Nov. 2004. Tracing the cosmological assembly of stars and supermassive black holes in galaxies. MNRAS 354, L37-L42.

Merritt, D., Sep. 2006. Mass Deficits, Stalling Radii, and the Merger Histories of Elliptical Galaxies. ApJ 648, 976-986.

Merritt, D., Milosavljević, M., Nov. 2005. Massive Black Hole Binary Evolution. Living Reviews in Relativity 8, 8-+.

Mihos, C., 1999. Dynamics of Mergers. Ap\&SS 266, 195-205.

Miralda-Escudé, J., Kollmeier, J. A., Jan. 2005. Star Captures by Quasar Accretion Disks: A Possible Explanation of the M- $\sigma$ Relation. ApJ 619, $30-40$.

Miyaji, T., Zamorani, G., Cappelluti, N., Gilli, R., Griffiths, R. E., Comastri, A., Hasinger, G., Brusa, M., Fiore, F., Puccetti, S., Guzzo, L., Finoguenov, A., Sep. 2007. The XMM-Newton Wide-Field Survey in the COSMOS Field. V. Angular Clustering of the X-Ray Point Sources. ApJS 172, 396405. 
Mo, H. J., Mao, S., White, S. D. M., Apr. 1998. The formation of galactic discs. MNRAS 295, 319-336.

Mo, H. J., White, S. D. M., Sep. 1996. An analytic model for the spatial clustering of dark matter haloes. MNRAS 282, 347-361.

Monaco, P., Fontanot, F., Taffoni, G., Mar. 2007. The MORGANA model for the rise of galaxies and active nuclei. MNRAS 375, 1189-1219.

Myers, A. D., Brunner, R. J., Nichol, R. C., Richards, G. T., Schneider, D. P., Bahcall, N. A., Mar. 2007a. Clustering Analyses of 300,000 Photometrically Classified Quasars. I. Luminosity and Redshift Evolution in Quasar Bias. ApJ 658, 85-98.

Myers, A. D., Brunner, R. J., Richards, G. T., Nichol, R. C., Schneider, D. P., Bahcall, N. A., Mar. 2007b. Clustering Analyses of 300,000 Photometrically Classified Quasars. II. The Excess on Very Small Scales. ApJ 658, 99-106.

Naab, T., Ostriker, J. P., Jan. 2009. Are Disk Galaxies the Progenitors of Giant Ellipticals? ApJ 690, 1452-1462.

Netzer, H., Trakhtenbrot, B., Jan. 2007. Cosmic Evolution of Mass Accretion Rate and Metallicity in Active Galactic Nuclei. ApJ 654, 754-763.

Overzier, R. A., Röttgering, H. J. A., Rengelink, R. B., Wilman, R. J., Jul. 2003. The spatial clustering of radio sources in NVSS and FIRST; implications for galaxy clustering evolution. A\&A 405, 53-72.

Padmanabhan, N., White, M., Norberg, P., Porciani, C., Feb. 2008. The real-space clustering of luminous red galaxies around $z<0.6$ quasars in the Sloan Digital Sky Survey. ArXiv:0802.2105.

Peng, C. Y., Ho, L. C., Impey, C. D., Rix, H.-W., Jul. 2002. Detailed Structural Decomposition of Galaxy Images. AJ 124, 266-293.

Peng, C. Y., Impey, C. D., Rix, H.-W., Kochanek, C. S., Keeton, C. R., Falco, E. E., Lehár, J., McLeod, B. A., Oct. 2006. Probing the Coevolution of Supermassive Black Holes and Galaxies Using Gravitationally Lensed Quasar Hosts. ApJ 649, 616-634. 
Pipino, A., Silk, J., Matteucci, F., Jan. 2009. Supermassive black holes, star formation and downsizing of elliptical galaxies. MNRAS 392, 475-482.

Porciani, C., Magliocchetti, M., Norberg, P., Dec. 2004. Cosmic evolution of quasar clustering: implications for the host haloes. MNRAS 355, 10101030 .

Porciani, C., Norberg, P., Oct. 2006. Luminosity- and redshift-dependent quasar clustering. MNRAS 371, 1824-1834.

Rafiee, A., Hall, P. B., Jan. 2009. Rapidly Spinning Black Holes in Quasars: An Open Question. ApJ 691, 425-430.

Rees, M. J., 1984. Black Hole Models for Active Galactic Nuclei. ARA\&A $22,471-506$.

Ross, N. P., Shen, Y., Strauss, M. A., Vanden Berk, D. E., Connolly, A. J., Richards, G. T., Schneider, D. P., Weinberg, D. H., Hall, P. B., Bahcall, N. A., Brunner, R. J., Jun. 2009. Clustering of Low-redshift $(z \leq 2.2)$ Quasars from the Sloan Digital Sky Survey. ApJ 697, 1634-1655.

Rovilos, E., Georgantopoulos, I., Nov. 2007. Optical colours of AGN in the extended Chandra deep field South: obscured black holes in early type galaxies. A\&A 475, 115-120.

Salpeter, E. E., Aug. 1964. Accretion of Interstellar Matter by Massive Objects. ApJ 140, 796-800.

Salucci, P., Szuszkiewicz, E., Monaco, P., Danese, L., Aug. 1999. Mass function of dormant black holes and the evolution of active galactic nuclei. MNRAS 307, 637-644.

Sanders, D. B., Soifer, B. T., Elias, J. H., Madore, B. F., Matthews, K., Neugebauer, G., Scoville, N. Z., Feb. 1988. Ultraluminous infrared galaxies and the origin of quasars. ApJ 325, 74-91.

Satyapal, S., Vega, D., Dudik, R. P., Abel, N. P., Heckman, T., Apr. 2008. Spitzer Uncovers Active Galactic Nuclei Missed by Optical Surveys in Seven Late-Type Galaxies. ApJ 677, 926-942. 
Sazonov, S. Y., Ostriker, J. P., Ciotti, L., Sunyaev, R. A., Mar. 2005. Radiative feedback from quasars and the growth of massive black holes in stellar spheroids. MNRAS 358, 168-180.

Scannapieco, E., Oh, S. P., Jun. 2004. Quasar Feedback: The Missing Link in Structure Formation. ApJ 608, 62-79.

Scannapieco, E., Silk, J., Bouwens, R., Dec. 2005. AGN Feedback Causes Downsizing. ApJ 635, L13-L16.

Schawinski, K., Thomas, D., Sarzi, M., Maraston, C., Kaviraj, S., Joo, S.-J., Yi, S. K., Silk, J., Dec. 2007. Observational evidence for AGN feedback in early-type galaxies. MNRAS 382, 1415-1431.

Seigar, M. S., Kennefick, D., Kennefick, J., Lacy, C. H. S., May 2008. Discovery of a Relationship between Spiral Arm Morphology and Supermassive Black Hole Mass in Disk Galaxies. ApJ 678, L93-L96.

Serber, W., Bahcall, N., Ménard, B., Richards, G., May 2006. The SmallScale Environment of Quasars. ApJ 643, 68-74.

Sesana, A., Vecchio, A., Volonteri, M., Apr. 2009. Gravitational waves from resolvable massive black hole binary systems and observations with Pulsar Timing Arrays. MNRAS 394, 2255-2265.

Seth, A., Agüeros, M., Lee, D., Basu-Zych, A., May 2008. The Coincidence of Nuclear Star Clusters and Active Galactic Nuclei. ApJ 678, 116-130.

Shankar, F., Bernardi, M., Jun. 2009. The age dependence of the size-stellar mass relation and some implications. MNRAS 396, L76-L80.

Shankar, F., Bernardi, M., Haiman, Z., Apr. 2009a. The Evolution of the $M_{B H}-\sigma$ Relation Inferred from the Age Distribution of Local Early-Type Galaxies and Active Galactic Nuclei Evolution. ApJ 694, 867-878.

Shankar, F., Cavaliere, A., Cirasuolo, M., Maraschi, L., Mar. 2008a. OpticalRadio Mapping: the Kinetic Efficiency of Radio-Loud AGNs. ApJ 676, 131-136.

Shankar, F., Crocce, M., Miralda-Escude', J., Fosalba, P., Weinberg, D. H., Oct. 2008b. On the radiative efficiencies, Eddington ratios, and duty cycles of luminous high-redshift quasars. ArXiv:0810.4919. 
Shankar, F., Dai, X., Sivakoff, G. R., Nov. 2008c. Dependence of the Broad Absorption Line Quasar Fraction on Radio Luminosity. ApJ 687, 859-868.

Shankar, F., Lapi, A., Salucci, P., De Zotti, G., Danese, L., May 2006. New Relationships between Galaxy Properties and Host Halo Mass, and the Role of Feedbacks in Galaxy Formation. ApJ 643, 14-25.

Shankar, F., Mathur, S., May 2007. On the Faint End of the High-Redshift Active Galactic Nucleus Luminosity Function. ApJ 660, 1051-1059.

Shankar, F., Salucci, P., Granato, G. L., De Zotti, G., Danese, L., Nov. 2004. Supermassive black hole demography: the match between the local and accreted mass functions. MNRAS 354, 1020-1030.

Shankar, F., Weinberg, D. H., Miralda-Escudé, J., Jan. 2009b. SelfConsistent Models of the AGN and Black Hole Populations: Duty Cycles, Accretion Rates, and the Mean Radiative Efficiency. ApJ 690, 20-41.

Shen, Y., Greene, J. E., Strauss, M. A., Richards, G. T., Schneider, D. P., Jun. 2008. Biases in Virial Black Hole Masses: An SDSS Perspective. ApJ 680, 169-190.

Shen, Y., Strauss, M. A., Oguri, M., Hennawi, J. F., Fan, X., Richards, G. T., Hall, P. B., Gunn, J. E., Schneider, D. P., Szalay, A. S., Thakar, A. R., Vanden Berk, D. E., Anderson, S. F., Bahcall, N. A., Connolly, A. J., Knapp, G. R., May 2007. Clustering of High-Redshift $(z \geq 2.9)$ Quasars from the Sloan Digital Sky Survey. AJ 133, 2222-2241.

Shen, Y., Strauss, M. A., Ross, N. P., Hall, P. B., Lin, Y.-T., Richards, G. T., Schneider, D. P., Weinberg, D. H., Connolly, A. J., Fan, X., Hennawi, J. F., Shankar, F., Vanden Berk, D. E., Bahcall, N. A., Brunner, R. J., Jun. 2009. Quasar Clustering from SDSS DR5: Dependences on Physical Properties. ApJ 697, 1656-1673.

Sheth, R. K., Bernardi, M., Schechter, P. L., Burles, S., Eisenstein, D. J., Finkbeiner, D. P., Frieman, J., Lupton, R. H., Schlegel, D. J., Subbarao, M., Shimasaku, K., Bahcall, N. A., Brinkmann, J., Ivezić, Ž., Sep. 2003. The Velocity Dispersion Function of Early-Type Galaxies. ApJ 594, 225231. 
Sheth, R. K., Mo, H. J., Tormen, G., May 2001. Ellipsoidal collapse and an improved model for the number and spatial distribution of dark matter haloes. MNRAS 323, 1-12.

Shields, G. A., Menezes, K. L., Massart, C. A., Vanden Bout, P., Apr. 2006. The Black Hole-Bulge Relationship for QSOs at High Redshift. ApJ 641, 683-688.

Silk, J., Rees, M. J., Mar. 1998. Quasars and galaxy formation. A\&A 331, L1-L4.

Silverman, J. D., Green, P. J., Barkhouse, W. A., Kim, D.-W., Kim, M., Wilkes, B. J., Cameron, R. A., Hasinger, G., Jannuzi, B. T., Smith, M. G., Smith, P. S., Tananbaum, H., May 2008. The Luminosity Function of X-Ray-selected Active Galactic Nuclei: Evolution of Supermassive Black Holes at High Redshift. ApJ 679, 118-139.

Small, T. A., Blandford, R. D., Dec. 1992. Quasar evolution and the growth of black holes. MNRAS 259, 725-737.

Soltan, A., Jul. 1982. Masses of quasars. MNRAS 200, 115-122.

Steed, A., Weinberg, D. H., Nov. 2003. Accretion Driven Evolution of Quasars and Black Holes: Theoretical Models. ArXiv Astrophysics eprints.

Tamura, N., Ohta, K., Ueda, Y., Jan. 2006. Supermassive black hole mass functions at intermediate redshifts from spheroid and AGN luminosity functions. MNRAS 365, 134-146.

Terlevich, A. I., Forbes, D. A., Mar. 2002. A catalogue and analysis of local galaxy ages and metallicities. MNRAS 330, 547-562.

Thacker, R. J., Scannapieco, E., Couchman, H. M. P., Richardson, M., Mar. 2009. Predictions of Quasar Clustering: Redshift, Luminosity, and Selection Dependence. ApJ 693, 552-563.

Thomas, D., Jul. 1999. Abundance ratios in hierarchical galaxy formation. MNRAS 306, 655-661. 
Thompson, T. A., Quataert, E., Murray, N., Sep. 2005. Radiation Pressuresupported Starburst Disks and Active Galactic Nucleus Fueling. ApJ 630, $167-185$.

Thorne, K. S., Jul. 1974. Disk-Accretion onto a Black Hole. II. Evolution of the Hole. ApJ 191, 507-520.

Tinker, J. L., Conroy, C., Norberg, P., Patiri, S. G., Weinberg, D. H., Warren, M. S., Oct. 2008. Void Statistics in Large Galaxy Redshift Surveys: Does Halo Occupation of Field Galaxies Depend on Environment? ApJ 686, 53-71.

Tremaine, S., Gebhardt, K., Bender, R., Bower, G., Dressler, A., Faber, S. M., Filippenko, A. V., Green, R., Grillmair, C., Ho, L. C., Kormendy, J., Lauer, T. R., Magorrian, J., Pinkney, J., Richstone, D., Aug. 2002. The Slope of the Black Hole Mass versus Velocity Dispersion Correlation. ApJ $574,740-753$.

Treu, T., 2004. The Formation of Early-type Galaxies: Observations to $z \sim 1$. In: Mulchaey, J. S., Dressler, A., Oemler, A. (Eds.), Clusters of Galaxies: Probes of Cosmological Structure and Galaxy Evolution. pp. 177-+.

Treu, T., Woo, J.-H., Malkan, M. A., Blandford, R. D., Sep. 2007. Cosmic Evolution of Black Holes and Spheroids. II. Scaling Relations at $\mathrm{z}=0.36$. ApJ 667, 117-130.

Tsujimoto, T., Aug. 2007. Fossil Imprints of Outflow from the Galactic Bulge in Elemental Abundances of Metal-rich Disk Stars. ApJ 665, L115-L118.

Tundo, E., Bernardi, M., Hyde, J. B., Sheth, R. K., Pizzella, A., Jul. 2007. On the Inconsistency between the Black Hole Mass Function Inferred from $M_{\odot}-\sigma$ and $M_{\odot}-L$ Correlations. ApJ 663, 53-60.

Ueda, Y., Akiyama, M., Ohta, K., Miyaji, T., Dec. 2003. Cosmological Evolution of the Hard X-Ray Active Galactic Nucleus Luminosity Function and the Origin of the Hard X-Ray Background. ApJ 598, 886-908.

Urrutia, T., Lacy, M., Becker, R. H., Feb. 2008. Evidence for Quasar Activity Triggered by Galaxy Mergers in HST Observations of Dust-reddened Quasars. ApJ 674, 80-96. 
Urry, C. M., Padovani, P., Sep. 1995. Unified Schemes for Radio-Loud Active Galactic Nuclei. PASP 107, 803-+.

Valluri, M., Ferrarese, L., Merritt, D., Joseph, C. L., Jul. 2005. The Low End of the Supermassive Black Hole Mass Function: Constraining the Mass of a Nuclear Black Hole in NGC 205 via Stellar Kinematics. ApJ 628, 137-152.

Vasudevan, R. V., Fabian, A. C., Nov. 2007. Piecing together the X-ray background: bolometric corrections for active galactic nuclei. MNRAS 381, $1235-1251$.

Vasudevan, R. V., Fabian, A. C., Jan. 2009. Simultaneous X-ray/optical/UV snapshots of active galactic nuclei from XMM-Newton: spectral energy distributions for the reverberation mapped sample. MNRAS 392, 11241140 .

Vestergaard, M., Feb. 2004. Early Growth and Efficient Accretion of Massive Black Holes at High Redshift. ApJ 601, 676-691.

Vittorini, V., Shankar, F., Cavaliere, A., Nov. 2005. The impact of energy feedback on quasar evolution and black hole demographics. MNRAS 363, $1376-1388$.

Volonteri, M., Madau, P., Quataert, E., Rees, M. J., Feb. 2005. The Distribution and Cosmic Evolution of Massive Black Hole Spins. ApJ 620, 69-77.

Wang, J. ., Hu, C., Li, Y. ., Chen, Y. ., King, A. R., Marconi, A., Ho, L. C., Yan, C. ., Staubert, R., Zhang, S., Apr. 2009. Episodic Random Accretion and the Cosmological Evolution of Supermassive Black Hole Spins. ArXiv:0904.1896.

Wechsler, R. H., Zentner, A. R., Bullock, J. S., Kravtsov, A. V., Allgood, B., Nov. 2006. The Dependence of Halo Clustering on Halo Formation History, Concentration, and Occupation. ApJ 652, 71-84.

Wetzel, A. R., Cohn, J. D., White, M., Apr. 2009. The clustering and host haloes of galaxy mergers at high redshift. MNRAS 394, 2182-2190.

White, M., Martini, P., Cohn, J. D., Nov. 2008. Constraints on the correlation between QSO luminosity and host halo mass from high-redshift quasar clustering. MNRAS 390, 1179-1184. 
Woo, J.-H., Treu, T., Malkan, M. A., Blandford, R. D., Jul. 2008. Cosmic Evolution of Black Holes and Spheroids. III. The $M_{B H}-\sigma_{*}$ Relation in the Last Six Billion Years. ApJ 681, 925-930.

Wyithe, J. S. B., Feb. 2006. A log-quadratic relation between the nuclear black hole masses and velocity dispersions of galaxies. MNRAS 365, 10821098.

Wyithe, J. S. B., Loeb, A., Oct. 2003. Self-regulated Growth of Supermassive Black Holes in Galaxies as the Origin of the Optical and X-Ray Luminosity Functions of Quasars. ApJ 595, 614-623.

Wyithe, J. S. B., Loeb, A., Dec. 2005. Constraints on the Process that Regulates the Growth of Supermassive Black Holes Based on the Intrinsic Scatter in the $M_{b h}-\sigma_{s p h}$ Relation. ApJ 634, 910-920.

Wyithe, J. S. B., Loeb, A., May 2009. Evidence for merger-driven activity in the clustering of high-redshift quasars. MNRAS 395, 1607-1619.

Yoo, J., Miralda-Escudé, J., Oct. 2004. Formation of the Black Holes in the Highest Redshift Quasars. ApJ 614, L25-L28.

Yoo, J., Miralda-Escudé, J., Weinberg, D. H., Zheng, Z., Morgan, C. W., Oct. 2007. The Most Massive Black Holes in the Universe: Effects of Mergers in Massive Galaxy Clusters. ApJ 667, 813-825.

Younger, J. D., Hopkins, P. F., Cox, T. J., Hernquist, L., Oct. 2008. The Self-Regulated Growth of Supermassive Black Holes. ApJ 686, 815-828.

Yu, Q., Lu, Y., Feb. 2004. Constraints on QSO Models from a Relation between the QSO Luminosity Function and the Local Black Hole Mass Function. ApJ 602, 603-624.

Yu, Q., Lu, Y., Dec. 2008. Toward Precise Constraints on the Growth of Massive Black Holes. ApJ 689, 732-754.

Yu, Q., Tremaine, S., Oct. 2002. Observational constraints on growth of massive black holes. MNRAS 335, 965-976.

Zhao, D. H., Mo, H. J., Jing, Y. P., Börner, G., Feb. 2003. The growth and structure of dark matter haloes. MNRAS 339, 12-24. 